\title{
Tracking multiple Autonomous Underwater Vehicles
}

\author{
José Melo ${ }^{1,2}$ (D) Aníbal C. Matos ${ }^{1,2}$ \\ Received: 2 May 2016 / Accepted: 12 January 2018 \\ (c) Springer Science+Business Media, LLC, part of Springer Nature 2018
}

\begin{abstract}
In this paper we present a novel method for the acoustic tracking of multiple Autonomous Underwater Vehicles. While the problem of tracking a single moving vehicle has been addressed in the literature, tracking multiple vehicles is a problem that has been overlooked, mostly due to the inherent difficulties on data association with traditional acoustic localization networks. The proposed approach is based on a Probability Hypothesis Density Filter, thus overcoming the data association problem. Our tracker is able not only to successfully estimate the positions of the vehicles, but also their velocities. Moreover, the tracker estimates are labelled, thus providing a way to establish track continuity of the targets. Using real word data, our method is experimentally validated and the performance of the tracker is evaluated.
\end{abstract}

Keywords Marine robotics · Position estimation · Underwater robotics $\cdot$ Multiple target tracking

\section{Introduction}

Autonomous Underwater Vehicles (AUVs) are becoming a reliable and cost-effective solution for performing a variety of underwater tasks in a fully automated way. Among the main tasks to be performed by AUVs are bathymetric surveys and environmental inspections, surveillance and patrolling, or even mine countermeasures operations. The use of such vehicles means that the assigned tasks can be performed usually in a cost-effective way, but also enables operations in challenging scenarios that otherwise would not be safe or even possible for human intervention. While most of these tasks are traditionally performed using only a single vehicle, there are significant research efforts focused towards the development of algorithms that allow fleets of AUVs, navigating in a coordinated fashion, to achieve a common goal.

The use of multiple vehicles allows the parallelization of tasks that otherwise would not be possible, thus reducing operations time. The potential for efficiency gains is even greater if the various vehicles are collaborating for the completion of a task. The use of teams of collaborating AUVs has

José Melo

jose.melo@fe.up.pt

Aníbal C. Matos

anibal@fe.up.pt

1 Faculty of Engineering, University of Porto, Rua Dr. Roberto Frias, s/n, 4200-465 Porto, Portugal

2 INESC TEC, Rua Dr. Roberto Frias, 4200-465 Porto, Portugal been foreseen for different applications, for example mine counter-measures missions (Prins and Kandemir 2008) or archaeological missions. Even though there is an extensive and growing literature on cooperative control theory, there are only a few approaches demonstrating complete multiAUV cooperation in field trials in water. An example of this would be the efficient mapping of a given area using multiple vehicles, addressed in (Paull et al. 2015). Teams of cooperating AUVs have also been reported to perform adaptive environmental sampling tasks, namely by having multiple vehicles performing plume tracking quickly and with high temporal and spatial resolution (Schulz et al. 2003). In a somewhat similar mission, sea trials with a fleet of ten autonomous underwater gliders deployed as an adaptive, coordinated ocean sampling network have also been reported (Fiorelli et al. 2006; Leonard et al. 2010). Within the framework of environmental sampling, a distributed multi-vehicle patrolling approach was also proposed (Marino et al. 2015).

With such developments, it is reasonable to expect that in a near future new applications will arise requiring the operation of multiple AUVs concurrently, cooperating to achieve a desired goal. With the increase of such multi-vehicle missions of underwater vehicles, the problem of tracking multiple AUVs in real-time becomes even more relevant. In fact, for most of the classical missions for AUVs, the ability to track the vehicles during operation time is not only desirable, but even critical for some more uncertain hazardous scenarios, such as the military or oil industry applications. 
Tracking AUVs can be done by listening to the acoustic signals exchanged between the vehicle and a set of acoustic beacons deployed in the area of operations. The AUVs need to emit an acoustic signal, that is then detected by each one of the beacons at different times, and according to their distance to the vehicle. By combining the Time-of-Flight (ToF) of the signals as detected by the beacons, the position of a vehicle can be computed by using multilateration techniques.

From the majority of the literature considering tracking of AUVs, only a few have been able to fully address the problem of tracking multiple vehicles. In fact, only seldom scenarios with multiple targets are considered and experimentally validated. The main obstacle in such cases is the ability to uniquely associate acoustic signals with the source that emitted such signals. Tracking more than one vehicle usually requires that each one of the vehicles emits signals that can be easily distinguishable between each other. A natural way to comply with this requirement is to have the vehicles using different frequency modulated signals. Alternatively, time-division multiplexing schemes can also been derived. While these approaches are proven, they are far from being optimal.

Actually, both options are hardly scalable, particularly when addressing situations with several vehicles. This is even more striking if, as frequently happens, the acoustic beacons are also required to emit their own acoustic signals, in order to provide navigational aids to the vehicles. When using time multiplexing schemes, time slots are attributed to each one of the devices operating on the network, so they can emit acoustic signals. For operations with multiple vehicles, the number of time slots is increased, which in turn also increases the time interval between two consecutive signal emission slots for a given vehicle. As the number of vehicles increases, this can significantly degrade the performance of the trackers. On the other hand, resorting to different frequency signals is also not a scalable approach. Increasing the number of distinct frequency signals is cumbersome and costly as it requires the development of specific hardware for emission and detection of the signals. This is even more complicated if we consider that the acoustic signals used are usually in a very confined band, approximately between around 10 to $30 \mathrm{kHz}$, which in turn limits the number of available frequencies.

\subsection{Contribution}

It can be argued that standard tracking algorithms, such as Kalman Filter based trackers, would be an appropriate solution for tracking multiple vehicles, as in the multiple AUV cooperative scenarios that have been mentioned. In fact, for most of the cases the number of vehicles in operation is known in advance, so it could be just the case of running multiple trackers in parallel. However, as it was made clear above, it is often challenging to have multiple vehicles emitting easily distinguishable acoustic signals. Therefore, in such scenarios standard tracking approaches would require, and struggle, with complex data-association methods.

In this article we are focused on a different approach to the problem of tracking multiple AUVs. In specific, we are trying to address the problem of tracking multiple AUVs that emit similar acoustic signals, which are not possible to distinguish between each other. The main contribution of this paper is then the derivation of a suitable algorithm for acoustically tracking multiple AUVs that can cope with such limitations. The proposed tracker, which is based on a Probability Hypothesis Density (PHD) filter, doesn't require any data association step, which means that it can estimate the positions and velocities of the different vehicles, even when they are emitting similar and otherwise undistinguishable acoustic signals. Besides that, an experimental validation of the proposed approach is also provided. Up to the authors knowledge, it is the first time that such an approach has been presented.

The work here presented is in fact an extension of a previous conference article (Melo and Matos 2014). That work was a preliminary study concerning the feasibility of using Random Finite Sets to address the problem tracking multiple AUVs. In a simulation environment, a PHD filter was considered for the problem of tracking three underwater autonomous vehicles using range-only acoustic measurements detected by an Long baseline (LBL) acoustic positioning network. Nevertheless that preliminary study was limited, in the sense that it did not fully address all the challenges of a realistic underwater AUV operations. For example, in the simulations presented all the vehicles had the same known and constant velocity, and only followed trajectories with a constant heading. These are obvious limiting assumptions. Moreover, multipath acoustic phenomenons, commonly observed in acoustic underwater communications, were not part of the simulation environment. At the same time, the proposed tracker had a tendency to overestimate the number of vehicles, which could potentially lead to incorrect target estimates. In what follows we will address these shortcomings of the initial approach.

In the work here presented there are no assumptions concerning the velocity or trajectory of the vehicles. One of the other major contributions of the work here presented is a suitable labelled formulation of the multitarget tracking problem, which enables the tracker to provide a full individual trajectory history of each of the individual targets. Another feature of interest provided is the ability to gracefully address situations with an unknown and time-varying number of targets. This is in fact a scenario that can be easily envisioned for future multi-vehicle operations, but it is not usually addressed by the traditional vector-based target tracking approaches. Another contribution here proposed is the derivation of a suitable deghosting heuristics that is able 
to deal with the emergence of ghost targets, as it will be made clear in the sections ahead. Finally, this article will also provided the experimental validation of the proposed algorithms, demonstrating the robustness of the tracker when in the presence of measurement ambiguity that characterizes underwater acoustic signals.

\subsection{Organization of the paper}

The paper proceeds as follows. Section 2 provides a review of work related to the topic of acoustic underwater target tracking. In Sect. 3 the single target tracking case is introduced. Details of the Finite Set Statistics framework that will be used, and derivation of the labelled Sequential Monte Carlo implementation of the PHD filter can be found, respectively, in Sects. 4 and 5. Section 6 details the experimental setup used to obtain data and validate this approach, while Sect. 7 presents the results obtained. Finally, in Sect. 8 we discuss the attained results and present some final remarks.

\section{Related work}

In this article we are focused on the problem of tracking multiple AUVs, which is of relevance for the majority of AUV operations. In this section we provide a brief state-of-the-art review of the topic of acoustic tracking of AUVs. However, tracking of AUVs can also be considered as part of the bigger, more general and broader area of tracking underwater acoustic sources. Research in this area has been investigated in other areas other than the AUV community, for example in the area of Underwater Wireless Sensor Networks (UWSNs). This will also be reviewed in this section. Nevertheless, the vast majority of the research so far has considered only the single target scenario.

Tracking of AUVs is perhaps the most well known application of underwater target tracking for the robotics community. The methods for tracking a single vehicle are well established and have been fairly addressed in the literature. On its essence, they all rely on computing the position of the vehicle from a set of acoustic ranges or bearings between a vehicle and a set of beacons, deployed at predefined fixed locations, whether at the surface or at the bottom of the sea. Examples of AUV tracking are found for instance by Watanabe et al. (2009), on which an accurate tracking method to estimate the position of AUV relative to a mother ship is described, using a Super-Short Baseline Navigation acoustic network. On the other hand, Melo and Matos (2008) demonstrate how tracking an AUV can be performed with an Inverted Long Baseline Navigation method with only two acoustic beacons. In line with the vast majority of the literature, both the references only address the problem of tracking a single AUV. Some commercially available AUV tracking systems pro- vide a way for simultaneously track multiple vehicles. One example of this is the ATACS system (Odell et al. 2002), which uses an identifier code transmitted with each signal, thus overcoming the problem of data association. This however, requires the existence of a data link between the nodes in the acoustic network. A different alternative is adopted by the GAPS systems (Napolitano et al. 2005), which makes use of different frequency modulated acoustic signals to be able to uniquely identify each of the nodes in the network.

Though not exactly the problem of tracking multiple vehicles, some closely related problems are also worth mentioning. For example, the problem of localizing multiple underwater acoustic sources using AUVs have been addressed in the literature. Choi and Choi (2015) address the problem of using a manoeuvring AUV equipped with several hydrophones to detect multiple acoustic sources, but considers only the case of a constant and known number of sources. Braca et al. (2014) propose the use of multiple AUVs as sensor nodes of a multistatic surveillance reconfigurable acoustic network with the purpose of detecting underwater targets in anti-submarine warfare environments. The use of UWSNs for acoustic tracking of underwater manoeuvring targets has also been addressed (Zhang et al. 2014; Wang et al. 2012). However, often the authors only consider the single target case.

\subsection{Tracking of multiple targets}

Only a few authors addressed problems of tracking multiple underwater targets, that are closely related to the one here in analysis. The problem of Multitarget Tracking in both multistatic active and passive sonobuouy systems has been addressed by Georgy et al. (2012), Georgy and Noureldin (2014). The authors detail a solution that is able to track an unknown time-varying number of multiple targets and keeping continuous tracks, in scenarios that depend on either bearing observations only, or both bearing and Doppler observations. Both papers present extensive simulation results, for scenarios with sixteen stationary receivers and two moving targets. On top of that, in the former, experimental results were also presented. The performance of several other multitarget trackers has also been assessed in similar environments, namely the Multi Target Tracker (MTT) (Lang et al. 2009) and a Gaussian-Mixture Cardinalized Probability Hypothesis Density Filter (GM-CPHD) (Georgescu et al. 2009; Erdinc et al. 2008), among others. In terms of the tools used, the latter work is closely related to the approach here proposed. In fact these two references share with the present work the use of algorithms based on the Random Finite Set (RFS) framework developed by Mahler (2008). Nevertheless, the underlying problem under study was the use of Multi-static sonar data for tracking a single moving source. 
Morelande et al. (2015) presented a method for detection and tracking of multiple underwater targets, in this case using a Sequential Monte Carlo (SMC) approximation of the Multiple Hypothesis Tracker (MHT), but only simulation results were presented. Kreucher and Shapo (2011) described a surveillance application on which a passive acoustic sensor array is used to monitor a given surveillance region and detect and track moving targets in the two dimensional space. The paper described a strategy to track multiples target using only bearing acoustic data from multiple passive arrays. First, a fixed discrete grid method is used for detection, while a particle filter is later used for tracking, This rather unique approach has been demonstrated using both simulated and real data. Despite its merits, the proposed Bayesian approach treats the problem, both conceptually and in implementation, as separate tasks of estimating the target present probability and the estimating target state probability. Moreover, aspects like missed detections and disappearing targets are not considered. Considering that, the present work proposes to use a RFS based approach, that is able to address those issues. Moreover, the detection and tracking problems are considered in an integrated way.

\section{Tracking a single AUV}

The scenario under analysis in this section is the one of tracking a single vehicle when using an LBL based system. This is done for the sake of clarity, as the concepts here presented also hold for the case of tracking multiple vehicles. In the remainder of this section we will briefly describe the working principles of LBL networks, and present the motion and sensor models used for the single target tracking problem. However, the interested reader should refer for example to the work by Melo and Matos (2008), and the references therein, for more depth in the topic.

Tracking an AUV requires it to be active, meaning that the vehicle needs to emit acoustic signals in order to be detected. The acoustic signals emitted by the AUV will then be detected by each of the acoustic beacons that compose the LBL acoustic network. LBL systems are one of the most robust, reliable and accurate configurations of Acoustic Navigation systems, and used in some of the most challenging scenarios, typically in the military and oil industries. Comparing to their counterparts, one of the main advantages of LBL systems is that they cover a relatively wide area and have very good, depth independent, position accuracy, which falls in the meter scale.

LBL systems need to have an array of acoustic beacons deployed on the seafloor, in specific predefined fixed location within the operation area. Alternatively, to overcome the need of deploying the beacons on the seafloor, the use of GPS-enabled intelligent buoys has been proposed, in a configuration called Inverted LBL. With the use of such systems,

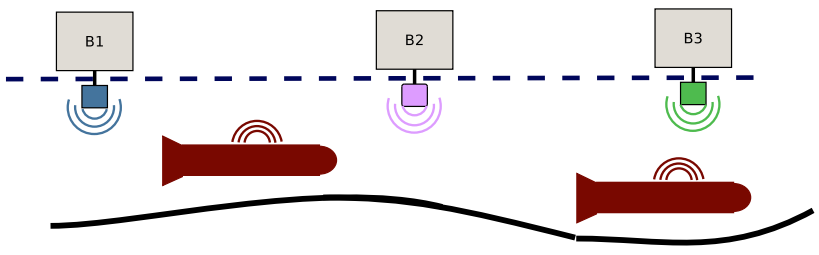

Fig. 1 Schematic view of and Inverted LBL setup for tracking multiple AUVs

the transponders of the bottom are replaced by floating buoys which carry the acoustic transducers, as exemplified in Fig. 1. Due to the fact that such devices also carry Global Navigation Satellite Systems (GNSS) receivers, calibration of such beacons can be significantly simplified. On the remainder of this article the focus will be on using GNSS enabled buoys as acoustic beacons. A detailed review of the different Acoustic Navigation schemes, their individual strengths and their disadvantages has been provided for example by Peñas (2009).

For the general case four beacons need to be deployed, in order to obtain the three dimensional position of the vehicle. However, under some specific conditions, for example when operating at shallow water and depths relatively small when comparing to the length of the baseline, this number can be smaller, as described by Melo and Matos (2008). In the remainder of this section we will only consider a set of two acoustic beacons, in an Inverted LBL configuration. Therefore, only the horizontal position of the AUV will be of interest.

With this setup, it is possible to directly compute ranges to each of the beacons from the ToF of the signals that each of the beacon detects. Figure 2 illustrates the scenario just described. The distances $d_{1}$ and $d_{2}$, which are, respectively, the slant ranges from the AUV to beacon 1 and beacon 2, are easily obtained from the time-of-flight of the acoustic signals. Then the position of the AUV can then be computed, by simple triangulation or multilateration of the ranges between the vehicle and all the beacons.

\subsection{Target and sensor model}

The system that was just described consists of an AUV navigating and periodically emitting an acoustic signal. These signals are detected by the two acoustic beacons, and then converted to range observations of the vehicle. Such scenario configures a typical target tracking scenario. The behaviour of the whole system can be described by the means of the single target dynamical model, $f_{k}$ and the single target measurement model h_k, (1) and (2), respectively.

$$
\begin{aligned}
& \mathbf{x}_{k}=f_{k}\left(\mathbf{x}_{k-1}, \mathbf{v}_{k-1}\right) \\
& \mathbf{z}_{k}=h_{k}\left(\mathbf{x}_{k}, \mathbf{n}_{k}\right)
\end{aligned}
$$




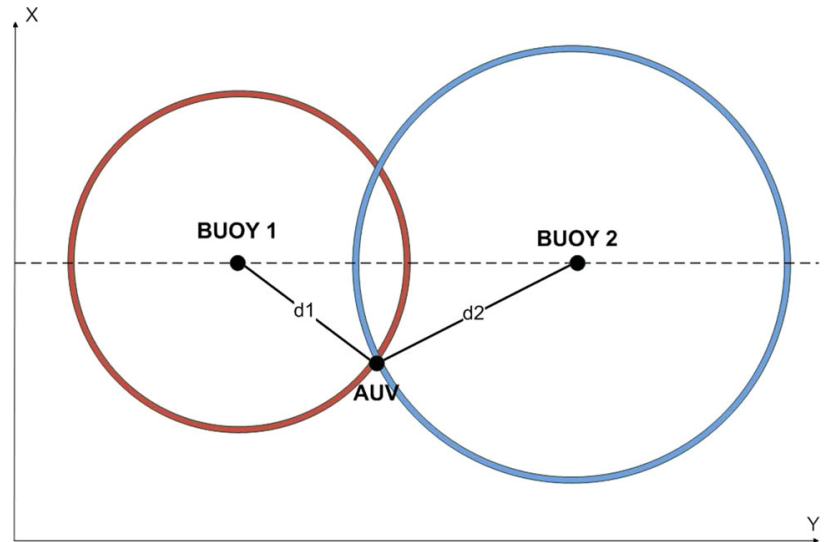

Fig. 2 Schematic view of the setup required for tracking AUVs using an LBL acoustic network

In the equations above, $\mathbf{x}_{k}$ refers to the state vector of a target. It is usually considered that the interesting state variables to be estimated are the vehicle's horizontal position and velocity. The state vector $\mathbf{x}_{k}$ can then be defined as:

$\mathbf{x}_{k}=\left[\begin{array}{llll}x_{k} & y_{k} & \dot{x}_{k} & \dot{y}_{k}\end{array}\right]^{T}$

Naturally, $x_{k}$ and $y_{k}$ denotes the position of the vehicle while $\dot{x}_{k}$ and $\dot{y}_{k}$ refer to the vehicle's velocity. It is further assumed that the dynamic model of the target follows a Gaussian constant velocity model, according to (4).

$$
\left[\begin{array}{c}
x_{k+1} \\
y_{k+1} \\
\dot{x}_{k+1} \\
\dot{y}_{k+1}
\end{array}\right]=\left[\begin{array}{llll}
1 & 0 & \Delta & 0 \\
0 & 1 & 0 & \Delta \\
0 & 0 & 1 & 0 \\
0 & 0 & 0 & 1
\end{array}\right]\left[\begin{array}{c}
x_{k} \\
y_{k} \\
\dot{x}_{k} \\
\dot{y}_{k}
\end{array}\right]+\mathbf{v}_{k}
$$

In the equation above $\Delta$ is the sampling interval and $\mathbf{v}_{k} \sim$ $\mathscr{N}(0, Q)$ is the white Gaussian process noise, with matrix $Q$ the process noise covariance. On a given sampling period interval, each of the acoustic beacons produces one range observation of the target, that is related with the state vector $\mathbf{x}_{k}$ according to the measurement equation:

$z_{k, i}=h_{i}\left(\mathbf{x}_{k}\right)+\mathbf{n}_{k, i}$

$h_{i}$ is the real valued function responsible for computing the expected range between the vehicle current position, and the location of each beacon, $\left(x_{0, i}, y_{0, i}\right)$, and $\mathbf{n}_{k, i} \sim \mathscr{N}\left(0, \sigma_{i}\right)$ is the measurement noise associated with acoustic beacon $i$, assumed to be Gaussian:

$h_{i}\left(\mathbf{x}_{k}\right)=\sqrt{\left(x_{k}-x_{0, i}\right)^{2}+\left(y_{k}-y_{0, i}\right)^{2}}$

A Bayesian estimation method is employed to estimate the position of the vehicle from the range measurements. Due to the non-linearity of the range measurements with respect to the system state, an Extended Kalman Filter is the most commonly used. However, the use of an Unscented Kalman Filter or even a Particle Filter would also be suitable.

Because the range observations are naturally noisy, with reflections and multipath phenomenons being detected by the acoustic transducers, outlier rejection strategies need to be employed. For this purpose, measurement gating can be done by comparing incoming ranges with normalized innovation squared (Matos et al. 1999; Fulton et al. 2000). A range measurement is only considered valid if

$v^{T} S^{-1} v<\gamma$

where $v=\left[z_{k}-F x_{x}\right]$ is the innovation vector and $S^{-1}$ corresponds to the innovation covariance matrix. The parameter $\gamma$ is given by an appropriate $\chi^{2}$ distribution, and it can be easily recovered from a distribution table for the desired confidence level. Alternatively, a suitable value can also be empirically determined.

Extending the single target case to accommodate the presence of multiple targets is not straightforward, mostly due to the difficulty of correctly distinguishing and associating acoustic signals emitted from each vehicle. In the next section we introduce the Random Finite Set framework, with which will be used to address exactly this problem.

\section{RFS and the PHD filter}

Multi-object filtering applications, such as the problem of tracking multiple vehicles, have been widely addressed, particularly by the radar tracking community. The objective of multi-object filtering is to jointly estimate the number of objects and their states from a set of observations. Both the Multiple Hypothesis Tracking (MHT) and the Joint Probabilistic Data Association (JPDA) have been presented in the literature as classical approaches for this problem (BarShalom and Li 1995; Stone et al. 2013). However, these traditional algorithms, rooted on the Bayes filtering paradigm present a number of pitfalls when addressing such scenarios.

Being based on the Kalman Filter, these algorithms rely on a vector representation, which requires stacking states and measurements from the different targets. This is not a satisfactory representation when both the number of targets and measurements are random and varying. Additionally, a data association step, on which an explicit associations between measurements and targets is established, is required, which can be computationally very demanding, or even intractable.

An alternative formulation for the multiple target estimation problem, and one that only recently emerged, can be achieved by using random set theory to formulate the general multisensor multitarget Bayes filter. On such approaches, both the collection of individual targets states and the collec- 


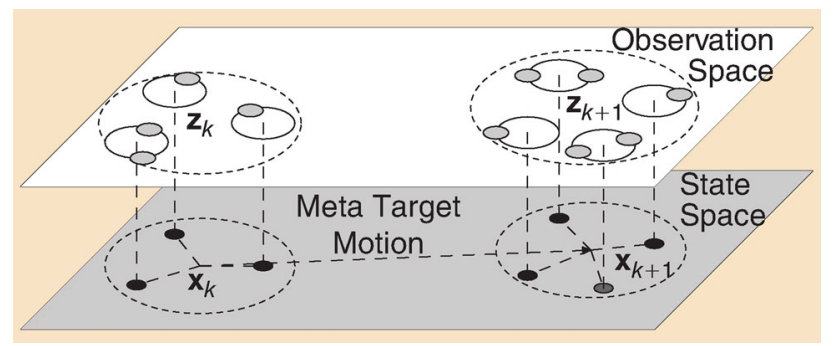

Fig. 3 Illustration the basic concept of FISST theory, according to which the multisensor-multitarget problem is transformed in a single "meta sensor - "meta target" problem (Granstrom et al. 2014)

tion of measurements are modelled as RFS, to obtain a set valued version of the general Bayes Filter. Loosely speaking, a RFS can be though of as a probabilistic representation of a collection of spatial point patterns that accounts for uncertainty in both the number of objects and their spatial locations. The usage of random finite sets, opposed to random vectors, is a more suitable formulation for addressing varying number of targets, target (dis)appearance and spawning, the presence of clutter and association uncertainty, false alarms and missed detections or even extended targets.

Developed by Mahler, the Finite Set Statistics framework (FISST) is a unified framework for data fusion based on random set theory, a geometrical and mathematically simplified version of point process theory. FISST provides a set of mathematical tools that allow direct application of Bayesian inferencing to multi-target problems (Vo et al. 2005). The aim of FISST is to transform multisensor-multitarget problems into single-sensor single-target problems, by bundling all sensors into a single "meta-sensor", all targets into a single "meta-target" and all observations into a single "metaobservation" (Mahler 2013). This is illustrated in Fig. 3. In that way, it is possible to construct true multisensormultitarget likelihood functions and true multitarget Markov transition densities from the motion models and measurement models of individual targets and sensors.

\subsection{Random finite set formulation of multitarget filtering}

Analogously to the traditional recursive single target Bayes Filter, the multisensor-multititarget Bayes Filter propagates the multitarget Bayes posterior density $p_{k}\left(\mathbf{X}_{k} \mid \mathbf{Z}_{1: k}\right)$ distribution through time, conditioned on the sets of measurements up to time $k, \mathbf{Z}_{1: k}$, using the traditional prediction-update recursion as follows,

$$
\begin{aligned}
& p\left(\mathbf{X}_{k} \mid \mathbf{Z}_{1: k-1}\right)=\int p\left(\mathbf{X}_{k} \mid \mathbf{X}\right) p\left(\mathbf{X}_{k-1} \mid \mathbf{Z}_{1: k-1}\right) \delta \mathbf{X} \\
& p_{k}\left(\mathbf{X}_{k} \mid \mathbf{Z}_{1: k}\right)=\frac{p\left(\mathbf{Z}_{k} \mid \mathbf{X}_{k}\right) p\left(\mathbf{X}_{k} \mid \mathbf{Z}_{1: k-1}\right)}{\int p\left(\mathbf{Z}_{k} \mid \mathbf{X}_{k}\right) p\left(\mathbf{X}_{k} \mid \mathbf{Z}_{1: k-1}\right) \delta \mathbf{X}}
\end{aligned}
$$

where the integrals present are set integrals, as introduced by the FISST framework.

In the recursion above (8) is the prediction step, while (9) is update step. Both the multitarget state transition function, $p\left(\mathbf{X}_{k} \mid X\right)$ and the multisensor likelihood function $p\left(\mathbf{Z}_{k} \mid \mathbf{X}_{k}\right)$ can be derived from the underlying single target single sensor and physical models using FISST techniques. Even though the general multisensor-multitarget Bayes filters in intractable for the general case, with the use of appropriate calculus tools introduced by FISST, it is possible to derive approximations, such as the Probability Hypothesis Density Filter.

Both the set of tracked objects $\mathbf{X}_{k}$ and the set of observations received $\mathbf{Z}_{k}$ at instant $k$ are modelled as random finite sets. It should be noted that, conversely to standard singletarget filtering, the dimensions of the random finite sets $\mathbf{X}_{k}$ and $\mathbf{Z}_{k}$ in the recursion (8-9) can vary with time.

$\begin{aligned} \mathbf{X}_{k} & =\left\{\mathbf{x}_{k, 1}, \ldots, \mathbf{x}_{k, M_{\mathbf{X}}(k)}\right\} \\ \mathbf{Z}_{k} & =\left\{\mathbf{z}_{k, 1}, \ldots, \mathbf{z}_{k, N_{\mathbf{Z}}(k)}\right\}\end{aligned}$

In (10) $M_{\mathbf{X}}(k)$ refers to the number of targets at instant $k$, while $N_{\mathbf{Z}}(k)$ in (11) refers to the the number of observations at the same instant.

The set of targets being tracked at a given time instant, $\mathbf{X}_{k}$, is composed by the collection of targets that survive from the previous time step, $\mathbf{S}_{k \mid k-1}$, together with the collection of spawned targets, $\mathbf{B}_{k \mid k-1}$, and the collection of new targets appearing only the in current time step, $\Gamma_{k}$.

$\mathbf{X}_{k}=\left(\bigcup_{\mathbf{x} \in \mathbf{X}_{k-1}} \mathbf{S}_{k \mid k-1}(\mathbf{x})\right) \cup\left(\bigcup_{\mathbf{x} \in \mathbf{X}_{k-1}} \mathbf{B}_{k \mid k-1}(\mathbf{x})\right) \cup \Gamma_{k}$

Similarly, the set of observations received at a given time instant (13) is a collection of both the measurements observed due to the present targets, $\Theta_{k}$, which also includes the probability of a missed detections, together with clutter measurements $\mathbf{K}_{k}$ corresponding to false alarms that may exist in that time instant.

$\mathbf{Z}_{k}=\mathbf{K}_{k} \cup\left(\bigcup_{\mathbf{x} \in \mathbf{X}_{k}} \Theta_{k}(\mathbf{x})\right)$

In fact, using random finite sets for modelling the multitarget state (12) and the multitarget measurements (13) provides an easy way to address target birth and spawning, or even high density of clutter measurements.

\subsection{PHD filter}

The Probability Hypothesis Density (PHD) Filter, initially proposed in Mahler (2003), is perhaps the most popular 
approximation to the optimal Bayesian multitarget filter. Instead of propagating the full posterior (9), the PHD filter propagates only the first-order statistical moment of the objects RFS of the objects. In a way, the PHD filter can be considered the multitarget counterpart of the constant gain Kalman Filter, that also only propagates the first order moment of a distribution.

Some assumptions must be observed in order to make the aforementioned propagation tractable, namely the signal to noise ratio (SNR) has to be high and all the targets should move independently of each other. The detection and measurement of a target is also assumed to be independent of other targets. Moreover, the PHD filter assumes that the RFS are Poisson RFS, one of the simplest class of RFS. The intensity function, also known in the tracking literature as the Probability Hypothesis Density, completely characterizes a Poisson RFS, thus the name of the filter. The interested reader should refer to Vo et al. (2005); Vo and Vo (2013) and the references therein for a theoretical insight on the foundations of the PHD filter.

A probability hypothesis density function is completely characterized by the property in (14), which means that integrating a given PHD function $\mathbf{D}_{k}$ over and entire region $S$ gives the expected number of objects in that region, $\hat{N}_{k \mid k}$. Additionally, the peaks of $\mathbf{D}_{k}$ identify the likely position of those objects:

$$
\int_{S} \mathbf{D}_{k \mid k}\left(\mathbf{X} \mid \mathbf{Z}^{(k)}\right) d \mathbf{X}=\hat{N}_{k \mid k}
$$

It should be noted that, as before, the integral present above is a FISST derived set integral.

The PHD filter consists on two equations: the predictor equation and the corrector equation, as follows. While the PHD filter predictor Eqs. (15-16) allow the current PHD to be predicted and extrapolated, the corrector equations (17-18) allow the predicted PHD to be updated with the observations (Mahler 2008).

$$
\begin{aligned}
& \mathbf{D}_{k \mid k-1}\left(\mathbf{x}_{k} \mid \mathbf{Z}_{1: k-1}\right)=\gamma_{k}\left(\mathbf{x}_{k}\right) \\
& \quad+\int \phi_{k \mid k-1}\left(\mathbf{x}_{k-1}\right) \mathbf{D}_{k-1 \mid k-1}\left(\mathbf{x}_{k-1} \mid \mathbf{Z}_{1: k-1}\right) d_{\mathbf{x}_{k-1}} \\
& \phi_{k \mid k-1}\left(\mathbf{x}_{k-1}\right)=p_{S, k}\left(\mathbf{x}_{k-1}\right) f_{k \mid k-1}\left(\mathbf{x}_{k} \mid \mathbf{x}_{k-1}\right)
\end{aligned}
$$

In the prediction equations above, $p_{S, k}$ refers to the probability a given target has to survive, from one time step to the following, $f_{k \mid k-1}$ refers to the single target state transition density, and $\gamma_{k}$ refers to the intensity of spontaneous births.

$$
\begin{aligned}
& \mathbf{D}_{k \mid k}\left(\mathbf{x}_{k} \mid \mathbf{Z}_{k}\right)=\Xi_{\mathbf{Z}_{k}}\left(\mathbf{x}_{k}\right) \mathbf{D}_{k \mid k-1}\left(\mathbf{x} \mid \mathbf{Z}_{k-1}\right) \\
& \Xi_{\mathbf{Z}_{k}}\left(\mathbf{x}_{k}\right)=\left(1-p_{D, k}\right)+\sum_{\mathbf{z} \in \mathbf{Z}_{k}} \frac{\psi_{k, \mathbf{z}}(\mathbf{x})}{\mathbf{K}_{k}+\left\langle\mathbf{D}_{k \mid k-1}, \psi_{k, \mathbf{z}}(\mathbf{x})\right\rangle}
\end{aligned}
$$

In the corrector equations above $p_{D, k}$ is the probability of detection of a target. $K_{k}$ refers to the false alarms clutter model, and $\langle\cdot, \cdot\rangle$ denotes the usual inner product. Additionally,

$\psi_{k, \mathbf{z}}(\mathbf{x})=p_{D, k} g(\mathbf{z} \mid \mathbf{x})$

with $g(\mathbf{z} \mid \mathbf{x})$ being the single target likelihood function.

\subsection{The sequential Monte Carlo PHD (SMC-PHD) filter}

A closed formed solution for the PHD filter (15-18) has been derived by Vo et al. (2005). This filter, the Gaussian Mixture PHD filter (GM-PHD) admits only scenarios on which the targets evolve and generates observations independently, and follow a linear gausian dynamical model. Additionally, is is also assumed that the sensors follow linear and Gaussian measurement models. Recalling the single target scenario described in Sect. 3, there are non-linearities present in the measurement model (2), thus this is not a suitable approach. Because of that, an approximation to the PHD filter recursion is more adequate.

The Sequential Monte Carlo PHD filter is an approximation to general PHD recursion that, analogously to standard Particle Filters, uses randomly distributed particles to approximate the density functions that represent the PHD predictor and corrector equations. For that reason, another possible designation for the SMC-PHD is Particle PHD filter. In fact, for the case when there is only one target with no birth, no death, no clutter and unity probability of detection, the PHD filter reduces to the standard particle filter.

Considering the particle approximation, the PHD predictor equation can be rewritten as in (20), where the approximation is done with $L_{k-1}$ particles, corresponding to the RFS containing the surviving targets, and $J_{k}$ new particles introduced, representing the RFS of the birth targets. For the general case the birth particles should cover the entire space of observation however, it is often the case that the prior knowledge regarding the location where possible new targets may appear is incorporated.

$\mathbf{D}_{k \mid k-1}\left(\mathbf{x}_{k}\right)=\sum_{i=1}^{L_{k-1}+J_{k}} w_{k \mid k-1} \delta_{\mathbf{x}_{k-1}^{(i)}}(\mathbf{x})$

where

$$
\mathbf{x}_{k \mid k-1} \sim \begin{cases}q_{k}\left(. \mid \mathbf{x}_{k-1}, \mathbf{Z}_{k}\right), & \text { if } 1 \leq i \leq L_{k-1} \\ p_{k}\left(. \mid \mathbf{Z}_{k}\right), & \text { if } L_{k-1}<i \leq L_{k-1}+J_{k}\end{cases}
$$


and

$w_{k \mid k-1}= \begin{cases}\frac{\phi_{k \mid k-1}\left(\mathbf{x}_{k-1}\right)}{q_{k}\left(\mathbf{x}_{k}^{(i)} \mid \mathbf{x}_{k-1}^{(i)}, \mathbf{Z}_{k}\right)} w_{k-1}, & \text { if } 1 \leq i \leq L_{k-1} \\ \frac{\gamma_{k}\left(\mathbf{x}_{k}^{i i)}\right)}{J_{k} p_{k}\left(\mathbf{x}_{k}^{(i)} \mid \mathbf{Z}_{k}\right)}, & \text { if } L_{k-1}<i \leq L_{k-1}+J_{k}\end{cases}$

where $q_{k}\left(. \mid \mathbf{x}_{k-1}, \mathbf{Z}_{k}\right)$ and $p_{k}\left(. \mid \mathbf{Z}_{k}\right)$ are two importance sampling proposal densities for the surviving and new born particles, respectively.

In the same way, the SMC approximation for the PHD corrector can be rewritten as in (23):

$\mathbf{D}_{k \mid k}\left(\mathbf{x}_{k}\right)=\sum_{i=1}^{L_{k-1}+J_{k}} w_{k \mid k} \delta_{\mathbf{x}_{k-1}^{(i)}}(\mathbf{x})$

where

$w_{k \mid k}=\left[\left(1-p_{D}\left(\mathbf{x}_{k}^{(i)}\right)\right)+\sum_{\mathbf{z} \in \mathbf{Z}_{\mathbf{k}}} \frac{p_{D}\left(\mathbf{x}_{k}^{(i)}\right) g_{k}\left(\mathbf{z}_{k} \mid \mathbf{x}_{k}\right)}{\mathbf{K}_{k}+c_{k}(\mathbf{z})}\right] w_{k \mid k-1}$

$\mathbf{K}_{k}$ refers to the intensity of the clutter measurement RFS and

$c_{k}=\sum_{i=1}^{L_{k-1}+J_{k}} p_{D}\left(\mathbf{x}_{k}^{(i)}\right) g\left(\mathbf{z}_{k} \mid \mathbf{x}_{k}\right) w_{k \mid k-1}$.

The particle transition density $f_{k \mid k-1}\left(\mathbf{x}_{k}^{i} \mid \mathbf{x}_{k-1}^{i}\right)$ and measurement likelihood $g\left(\mathbf{z}_{k} \mid \mathbf{x}_{k}\right)$ are obtained reusing the previously derived single target dynamical model (4) and measurement model (5), respectively. However, it should be noted that the measurement gating strategy adopted for the single target case (7) is not needed. Further details on the derivation and convergence properties of the SMC-PHD filter can be found in (Vo et al. 2005; Clark 2006)

Following the prediction and correction stages of the SMC-PHD filter, and analogously to what happens with the standard particle filter, there is a need to resample the particles in order to prevent sample impoverishment. Though different resampling strategies can be applied, such as stratified sampling or residual sampling, there is a common preference towards the use of systematic resampling, since this algorithm is easy to implement, has a linear computational complexity and, from a uniform distribution perspective, is theoretically superior (Hol et al. 2006).

The resampling stage of the SMC-PHD filter differs only from the traditional resampling strategies adopted in standard particle filters in that in the PHD filter the weights are not normalized to sum up to one, but instead,to the total particle mass $\hat{N}_{k \mid k}$ (Clark 2006), where

$\hat{N}_{k \mid k}=\sum_{i=1}^{L_{k-1}+J_{k}} w_{k \mid k}^{(i)}$

Because in the prediction stage of the algorithm there are always a number $J_{k}$ of birth particles that are introduced, the number of particles is always increased on every time step of the filter. Therefore, in the resampling stage of the PHD filter the number of particles in downscaled proportionally to the expected total number of targets, which is given by the nearest integer of the to the total particle mass, int $\left(\hat{N}_{k \mid k}\right)$.

Though not a integral part of the original SMC-PHD algorithm, target estimation plays an important role as it is the step where the locations of each of the targets are obtained. One way to perform this is to estimate the number of present targets in the current time-step, and then perform k-means clustering. Another alternative would be to fitting a Gaussian Mixture Model to the particles of the current time-step. While in principle any general clustering techniques could be employed, there has been a strong preference of the community on using the two methods mentioned.

\section{Tracking multiple AUVs}

Previous preliminary work demonstrated the suitability of a PHD filter to the problem of tracking multiple AUVs using range-only observations Melo and Matos (2014). There, simulation results validated the proposed approach to track multiple vehicles. However, and as discussed in Sect. 1, the aforementioned work was only a preliminary study on the topic, supported only by elementary simulations. In fact, such work dealt only with linear vehicles moving trajectories, not providing features such as track labelling or velocity estimation of the different vehicles. In this article we present a natural extension of that work, complementing it in various ways. This section is devoted to provide the details of such refinements to the original PHD filter, providing new features and making it more robust to real world applications.

\subsection{Observation set}

From the corrector equations of the SMC-PHD filter (23-25), the measurement model $g\left(\mathbf{z}_{k} \mid \mathbf{x}_{k}\right)$ stems directly from the single target measurement model (5). However, the elements $z_{k, i}$ of the measurement set $\mathbf{z}_{k}$, have a slightly more intricate formulation. Because there is no association between detected signals and targets, all the ranges detected by the beacons need to be combined, in order to accommodate an adequate observation set. 
At a given time step, each of the beacons will have a random number of detections, that are the result of the acoustic signals emitted by the vehicles, but also from possible clutter measurements that might exist. Thus, for a given beacon $j$, its corresponding detections during time period $k$ will be

$b_{k}^{j}=\left\{r_{k, 1}^{j}, \ldots r_{k, u}^{j}\right\}$

where $r_{k, u}^{j}$ is the $u$-th range detected by beacon $j$ at time $k$. The measurement set $\mathbf{z}_{k}$ will then consist off all the possible combinations of the detections by every beacon. In that way, and considering only two beacons as previously specified, the $i$-th element of the measurement set $\mathbf{z}_{k}$ will then simply be

$z_{k, i}=\left[r_{k, u}^{1} r_{k, v}^{2}\right]^{T}$,

and $\left|\mathbf{z}_{k}\right|$, the number of elements or cardinality of the set $\mathbf{z}_{k}$ will be $u \times v$. This process is then of combinatorial nature, which can present some problems if the number of beacons is very high. However, this is not likely to be the case, due to the particular conditions of our application.

The intensity of clutter measurements $\mathbf{K}_{k}$ is modelled as a Poisson RFS uniformly distributed over the surveillance region as

$\mathbf{K}_{k}=\lambda_{k} V u(z)$,

where $\lambda_{k}$ is the average number of clutter returns per unit volume, $V$ is the volume of the surveillance region, and $u(z)$ is the uniform density over the surveillance region. $\lambda_{k}$ will be varying over time, and dependent on the number of targets navigating. The considered average number of clutter measurements present is proportional to $(\# A U V)^{b}-1$, where $b$ is the number of beacons.

\subsection{Track labelling}

In multitarget applications it is often necessary not only to estimate the position of multiple objects, but also to estimate their paths, or trajectories. In order to do so, it is frequent to attribute a unique label to each target, so that each label is consistently associated with the same target over time. However, the formulation of the standard PHD filter, provided in the previous sections, gives no information on the track, meaning that there is no association between the estimated targets on a given time step, to the ones on the previous step.

In the literature some approaches to track labelling issue in standard PHD filters have already been proposed. Clark and Bell (2007) proposed two alternative labelling methods, one based on assigning labels to individual particles of the SMC-PHD filter, and the other and estimate-to-track method that finds the best association between estimated states and the predicted estimate derived from projecting the previous estimates with the motion model. Similarly to the latter, Lin et al. (2006) presented a track-labelling strategy on which the association between tracks and labels is based on a optimization problem which aims to minimize the cost of associating the peaks to tracks. A labelling solution by state augmentation was proposed by Ma et al. (2006), for the particular case of multitarget, where at most one target is allowed to be born at one time. This simple strategy of adding a track label was also demonstrated to help on the state estimation process. Based on this work, a further refinement was made to address the general RFS multiobject tracking scenario, with the concept of Labelled RFS being introduced in (Vo and Vo 2013).

A similar approach will be followed here, on which the state vector, $\xi_{k}$, was augmented by a variable, $\gamma_{k}$, to indicate the track identity as follows:

$\mathbf{x}_{\mathbf{k}}=\left[\xi_{k}^{T}, \gamma_{k}\right]^{T}$

Naturally, this transformation of the state vector also requires a convenient modification of the process model for the surviving particles (4), considering that the target label remains constant:

$\mathbf{x}_{k+1}=\left[\begin{array}{cc}A & 0 \\ 0 & 1\end{array}\right] \mathbf{x}_{k}+\left[\begin{array}{l}B \\ 0\end{array}\right] \mathbf{w}_{k}$

With the notation introduced, the use of clustering algorithms for state target estimation is no longer needed. Making use of the label variable introduced, target estimation can be computed simply by aggregating all the particle with the same label, as will be detailed further ahead. This is of particular relevance in SMC implementations of the PHD filter.

\subsection{Refinement of PHD filter}

Recalling from the previous sections, we are limited to the use of a SMC implementation of the PHD filter due to the non-linearities present in the measurement model. As other standard Monte Carlo methods, the SMC-PHD filter also suffers from the curse of dimensionality (Vo et al. 2005). In fact, SMC implementations are known to grow exponentially with the dimension of the state vector, therefore it is interesting to keep the dimensions of the state vector to a minimum. Otherwise, the number of the particles would have to grow significantly in order to prevent the available data to become sparse. This is of particular relevance when addressing multiple target tracking scenarios, as the number of vehicles being tracked can grow significantly.

Recalling from the previous subsection, it was chosen to augment the state vector with a variable to for track labelling. However, this will increase the dimension of the state vector. 
In order to keep the complexity of the filter low, and cope with this requirement, we introduce the following refinements to the PHD filter.

Picking up on the predictor equations of the SMC-PHD filter (20-22) and recalling that

$\phi_{k \mid k-1}\left(\mathbf{x}_{k-1}\right)=p_{S, k}\left(\mathbf{x}_{k-1}\right) f_{k \mid k-1}\left(\mathbf{x}_{k} \mid \mathbf{x}_{k-1}\right)$,

we redefine the single-target dynamical model, presented in Sect. 3 , to include the target label, $I_{k}^{i}$. In addition, we choose to include in the system state only the targets position on the horizontal plane, $x$ and $y$, so that the state vector is kept to a minimum dimension.

Therefore we define $\mathbf{x}_{k}^{i}=\left[x_{k}^{i}, y_{k}^{i}, \mathbf{I}_{k}^{i}\right]^{T}$. Naturally, the index $i$ refers to the target $i$, while $k$ refers to the time instant. With this definition, the target motion model becomes

$\mathbf{x}_{k+1}=\mathbf{x}_{k}+\left[\begin{array}{c}v_{x_{i, k}} \Delta \\ v_{y_{i, k}} \Delta \\ 0\end{array}\right]+v_{k}$

with the quantities $v_{x_{i, k}}$ and $v_{y_{i, k}}$ corresponding to the average velocities of the different targets, and $\Delta$ to the length of the time step. The different velocities of each target will be estimated once the position of the targets is estimated, as it will be detailed in the next subsection.

The general SMC-PHD filter assumes that new targets can be birthed across the entire observation space. Though a convenient assumption, this means a huge number of newborn particles must be drawn from a uniform density across the whole surveillance area. In this implementation an alternative path was chosen, as it is reasonable to assume that for applications where multiple AUVs are used, the positions from where the vehicles are usually launched in the water are known. For this reason it was assumed that new targets can appear spontaneously according to a Poisson Point Process with intensity function $\gamma=\mathscr{N}\left(. ; \mathbf{x}_{\gamma}, Q_{\gamma}\right)$ where $\mathbf{x}_{\gamma}$ and $Q_{\gamma}$ represent the centre and variance of the location where AUVs are launched.

\subsection{Velocity estimation}

Estimating the velocity of targets will have a paramount relevance in the performance of the tracker. The velocity will be important on the propagation of the particles that correspond to each of the targets on the most accurate direction, but also on preventing the appearance of ghost targets. Once the position estimates for each of the active targets has been computed, we can estimate the velocities. We do this by using Least Square Estimator (LSE) with forgetting factor. The advantages of using an additional estimator for the velocity, instead of augmenting the state vector detailed above, are mostly in terms of computational complexity. Additional, it is likely that by using the LSE results in smoother and less noisy velocity estimates.

We consider that all the vehicles move in straight lines, and their movement in both $x$ and $y$ directions can be independently described by the following equations:

$$
\begin{aligned}
& x(t)=x_{0}+v_{x} t \\
& y(t)=y_{0}+v_{y} t
\end{aligned}
$$

In (33), $x(t)$ and $y(t)$ are the current targets positions, while $t$ is naturally the time instant. On the other hand, $x_{0}, y_{0}, v_{x}$ and $v_{y}$ are the parameters to be estimated.

The estimation of the velocity occurs in three different moments. Whenever a target is first detected there is no prior information about its direction or speed, therefore random velocities are assumed in both the directions:

$v_{i, x}, v_{i, y} \sim \mathscr{N}\left(v_{r e f}, \sigma_{r e f}\right)$

In a second moment, when there is already a window of a number $w$ of previous position estimates of a given target, the parameters are estimated using the general LSE estimator, where $\theta$ is the vector of parameters to be estimated and $X$ and $Y$ are the vectors of model variables and observations, respectively. This calculation provides the first estimation of the velocities of the targets.

$\theta=\left(X^{T} X\right)^{-1} X^{T} Y$

On a third moment, we implement a Recursive LSE (RLSE) with forgetting factor. The RLSE with forgetting has been widely used in estimation and tracking of timevarying parameters in various fields of engineering (Vahidi et al. 2005). Not only the RLSE requires less computational power, but the use of a forgetting factor is more appropriate for estimating time-varying parameters, providing somewhat smoother estimates with less delay, as more weight is given to more recent observations. The RLSE can be implemented with the following equations:

$$
\begin{aligned}
\theta_{k} & =\theta_{k-1}+L_{k}\left(y_{k}-\phi_{k}^{T} \hat{\theta}_{k-1}\right) \\
L_{k} & =P_{k-1} \phi_{k}\left(\lambda+\phi_{k} P_{k-1} \phi_{k}\right)^{-1} \\
P_{k} & =\left(I-L_{k} \phi_{k}^{T}\right) P_{k-1} \frac{1}{\lambda}
\end{aligned}
$$

The RLSE, in (36), presents a similar structure to the Kalman Filter; it consists on the equations that recursively compute the parameters $\theta_{k}$, the gain, $L_{k}$ and the covariance, $P_{k}$.

\subsection{Target estimation}

Recalling from the previous sections, it was chosen to augment the state vector with a state variable for track labelling 
purposes. Doing so simplifies a great deal the target estimation step, which then can be reduced to aggregating the all the particles with the same label, and computing its average position. That is, for a given target with label 1 , its state $\xi_{k}$ can be recovered as follows:

$\hat{\xi}_{k}=\frac{1}{N_{k}\left(\mathbb{R}^{n}, 1\right)} \int \xi_{k} N_{k}\left(d \xi_{k} ; 1\right)$

that is, expected state vector of the track $1, \hat{\xi}_{k}(1)$ at time $k$, conditioned on the hypothesis that the track $I$ is present in that time instant. $N_{k}(:, 1)$ is the number of times that the track $I$ is present at time $k$.

In the specific case of the SMC-PHD filter, the fact that the information about each of the targets label is now incorporated in the state vector of every particle simplifies a great deal the task of target estimation. Obtaining the particles tracking a particular target resorts only to gather all the particles than have a specific label. From there, the target state of a particular target can be estimated as in an analogous way to the traditional particle filters:

$\hat{\xi}_{k}(1)=\frac{1}{\hat{N}_{k}(l)} \sum_{i=1}^{L_{k}} w_{k}^{i} \mathbf{1}_{\mathbf{x}_{k}}\left(\gamma_{k}^{i}=1\right) \xi_{k}^{i}$

where $\mathbf{1}_{A}$ is the indicator function, or characteristic function, defined on a set $\mathbf{X}$ that indicates the membership of an element in a subset $\mathbf{A}$ of $\mathbf{X}$, as follows

$\mathbf{1}_{A}(x):= \begin{cases}1 & \text { if } x \in A, \\ 0 & \text { if } x \notin A .\end{cases}$

The number of targets estimated by filter is given by the sum of all the weights, as in the particle filter. Additionally, a target with label 1 is estimated to be present if the sum of weights of the particles with associated with that label is above a certain threshold, usually defined as 0.5

$\hat{N}_{k}(1)=\sum_{i=1}^{L_{k}} w_{k}^{i} \mathbf{1}_{\mathbf{x}_{k}}\left(\gamma_{k}^{i}=1\right)$

It is also on the Target Estimation step that newborn particles are promoted to new targets. All the particles without any label are summed and, if they are above a given threshold, they are promoted to a new target and a new label is assigned. Despite the simplicity of the target estimation process, some care needs to be taken in order to prevent undesirable situations, for instance the appearance of ghost targets. This is particularly relevant since the resampling step of the SMCPHD filter is agnostic to labels. This will be further detailed in the next subsection.

\subsection{Deghosting}

The position of the targets can be computed by using multilateration techniques, that combine range measurements observed by different acoustic beacons. However, combining observations that are originated from disparate targets can generate a ghost target. In multiple target tracking scenarios involving multiple sensors, and particularly in multilateration applications, the appearance of ghost targets is recurrent. This happens because observations are naturally unlabelled, thus it is not possible to establish from which target they have been originated. It is therefore very important to be able to disambiguate between real targets and ghost targets. Deghosting is the name give to the different techniques that are used to distinguish and removing ghost targets from true targets. In the literature, different approaches have been suggested (Yang et al. 2013; Mazurek 2008).

In our specific application, ghost targets are likely to arise whenever two or more targets are equally distant from one of the acoustic beacons. Because it is not possible to distinguish between the acoustic signals emitted by each of the vehicles, from that point onwards it is likely that a ghost target arises. Based on empirical evidence, a heuristic deghosting approach has been implemented.

The followed strategy is based on monitoring the particle divergence for each target, $\Sigma_{1, k}$. If a ghost target arises, then the particles following a given target will divide and diverge, with a group of particles tracking the real target, and another group of particles tracking the ghost target. Therefore, if the divergence of the particles is above a certain threshold, then action is needed in order to prevent the appearance of a ghost target.

The implemented heuristics uses a k-means clustering algorithm to identify and partition the particles into two different clusters, $P_{1, k}^{1}$ and $P_{1, k}^{2}$. The two partitions will then be compared, with the partition with the highest cumulative weight assigned to the true target, and the other one corresponding to the ghost target. Consequently, the particles associated with the ghost target will be disregarded, while the particles tracking the true targets will be resampled to the number of particles per target, $N_{p}$. The implemented heuristics is detailed in Algorithm 1.

\subsection{Implementation}

As a summary of this section, we provide in Algorithm 2 the pseudo-code for the entire tracker for multiple AUVs. The recursive algorithm can be informally described by the different stages: particle prediction, measurement correction, resampling step, target estimation and velocity estimation. On the prediction stage, each of the $L_{k-1}$ particles that survived from the previous time step is propagated according the multitarget state transition density, and additional $J_{k}$ birth 


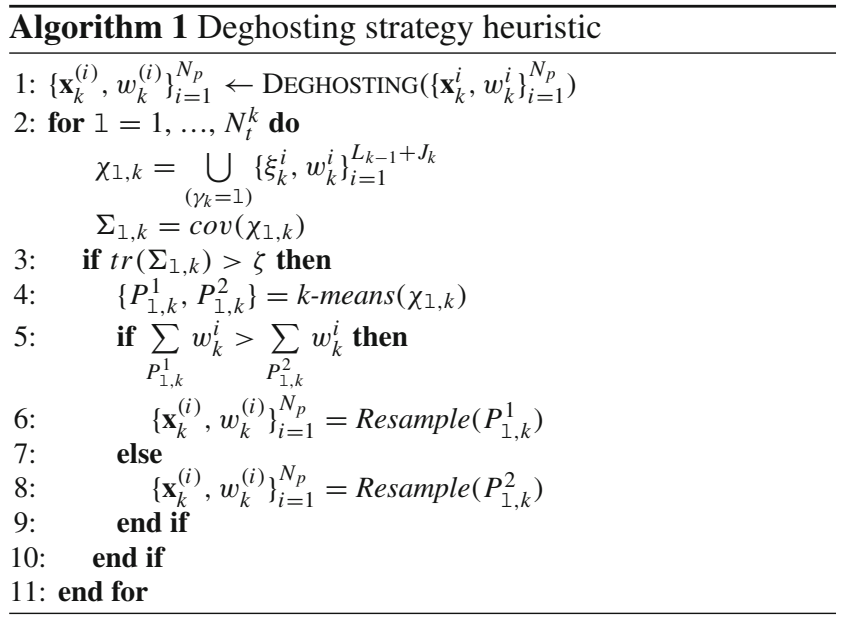

particles are introduced. Following the prediction stage, in the corrector stage all the particles are weighted according to the multisensor likelihood. After that, the resampling step takes place, where particles with low weights are replaced by copies of the particles with higher weights. Finally, the targets positions can be estimated from the set of resampled particles, and from such position estimates the individual velocities of each target is computed.

\section{Field trials}

The work presented is this article is devoted to the development of a tracker that is able to acoustically track multiple AUVs navigating simultaneously. In this section, we provide the details and document a series of field trials that allowed the experimental evaluation of the tracker. Those field trials were performed in February 2015 in the Douro river, a few kilometres upstream from Porto, in Portugal. In those trials it was possible to collect data for the experimental validation of the multiple AUV tracker derived in the previous sections, and the results of it will presented in this section.

The ideal setup for the experimental validation of the proposed algorithm would consist, naturally on a set of buoys or acoustic beacons, and a set of multiple AUVs naviga-

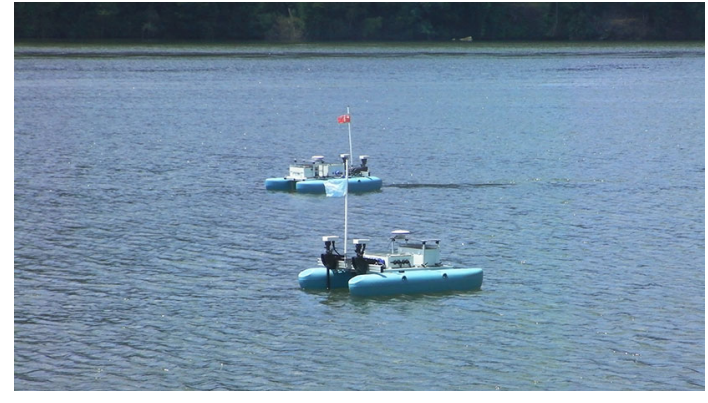

Fig. 4 The two ASVs, used as AUV surrogates in the field trials

tion in an open-water scenario. However, with such setup it would be hard to compare the performance of the filter tracking the multiple vehicles, due to the lack of the necessary ground-truth data. In order to overcome this, in the configuration under analysis it was chosen to replace the AUVs with Autonomous Surface Vehicles (ASVs), instead of AUVs. The ASVs can mimic the behaviour of AUVs if they are equipped with an acoustic transducer that always remains underwater, and at a constant depth. By doing we can use them as AUV surrogates. On the one hand this allows to have access to a series of acoustic underwater slant ranges obtained between moving vehicles and the respective beacons. On the other hand, because the ASVs are equipped with GPS receivers, we can have access to GPS derived ground truth data for the position and velocity of the vehicles.

In these trials we used two ASVs, namely the ASVs Gama and Zarco, depicted in Fig. 4. Gama and Zarco are two small sized catamaran based craft, designed to operate in quiet waters, and can reach speeds of up to $2 \mathrm{~m} / \mathrm{s}$. These vehicles can be remotely operated or autonomously perform pre-programmed missions. The vehicles are equipped with a set of navigation instruments, including a high-precision GPS receiver, which provide an accurate positioning level, a WiFi link for real-time connection with shore, and the necessary acoustic transceiver. For more details regarding these vehicles, the interested reader should refer to Cruz et al. (2007)

While traditionally a minimum of four beacons are needed to operate in an LBL acoustic network, it is possible to use fewer beacons as long as some assumptions are made. In

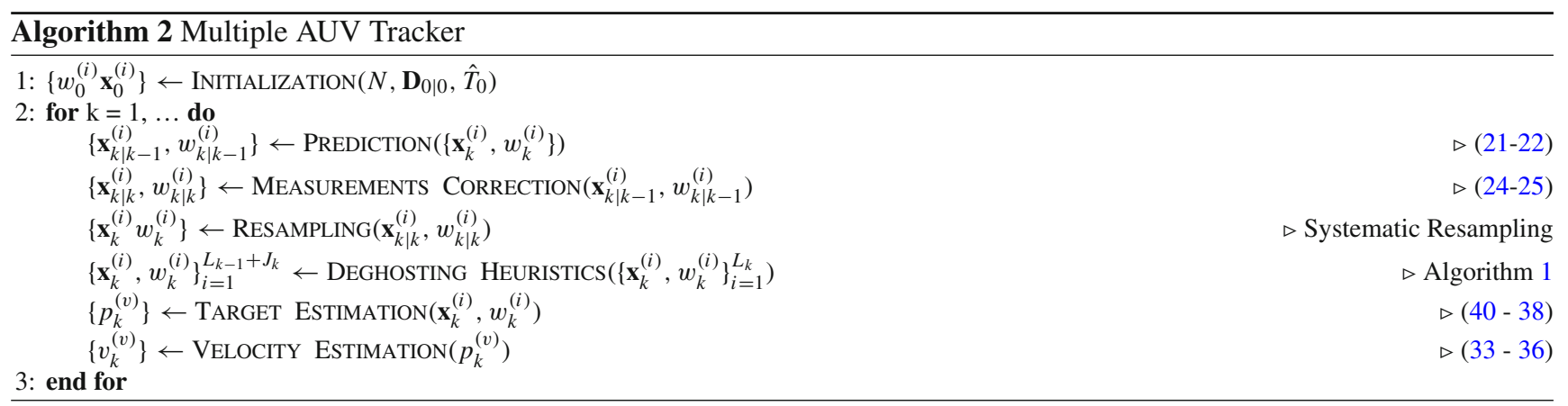


specific, if the vehicles are operating in very shallow waters, when compared to the distance of the baseline, and additionally, if it is guaranteed that the vehicles only operate in one side of the baseline, then it is possible to use only two beacons. In that case, it is only possible to retrieve the horizontal position of the targets.

In the field trials here in analysis set of two acoustic beacons floating at the surface, also referred to as buoys, were also used in an Inverted LBL configuration. Nevertheless, the underlying principles and the obtained results still apply whenever additional acoustic beacons are used.

We assume that the clocks sources of both the vehicle and the set of buoys are synchronized and with drifts that are small enough, so that One-Way-Travel-Time (OWTT) techniques can be used throughout the entire duration of the missions without any major concerns. What this assumption means is that all the systems, buoys and vehicle, share a common clock source and are aware of the exact time instant each of the systems emits a given acoustic signal. For moderate operation durations, up to a few hours, clock synchrony can be achieved by a combination of a GNSS receiver and the Network Time Protocol (NTP). As it will be further detailed in the following sections, this is in fact the solution used for the field trials here presented. Together with UTC time, some GNSS receivers are also able to provide a Pulse-Per-Second (PPS) signal, synchronous with UTC time, that can be used for clock discipline purposes. By combining that with one of the available implementations of the NTP protocol, it is possible to maintain synchrony even when the GNSS receiver fails, for example vehicles submerge and operate underwater. For long-term operations, a more stable clock source might be required.

We are only considering the acoustic signals emitted by the AUVs, synchronously and at a frequency of $1 \mathrm{~Hz}$. Considering a speed of sound in the water of approximately $1500 \mathrm{~m} / \mathrm{s}$, this restricts the operations into an area of around $1500 \mathrm{~m}$ of distance to each of the buoys, a fairly mild assumption for shallow water missions. With this setup it is possible to directly compute ranges to each of the beacons from the ToF of the signals that each of the beacon detects.

\subsection{GPS measurements}

All the devices used, beacons and vehicles, are equipped with GNSS receivers which provide accurate position data throughout the duration of the trial. This data will serve as ground truth of the whole experiment, and will be compared with the position of the targets estimated by the tracker. Therefore, is of utmost importance to understand how the variance of the GPS position measurements can affect the results obtained.

While it is assumed that the position of the beacons remains the same, that is not necessary accurate. Even though
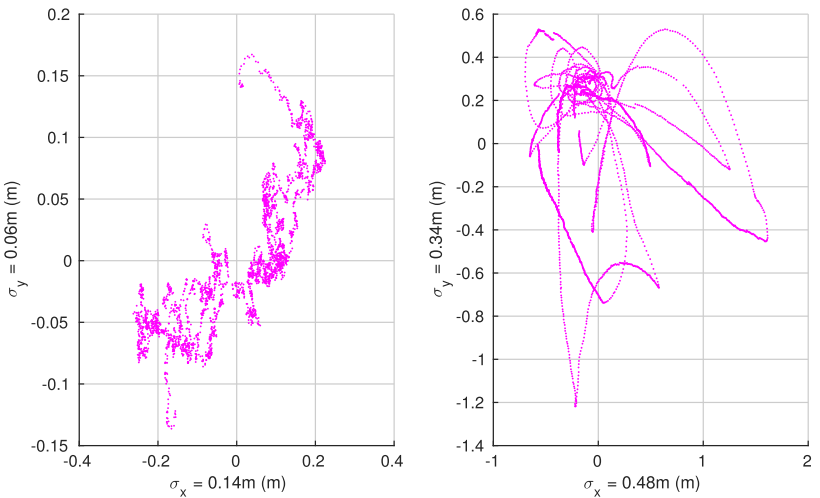

Fig. 5 Dispersion of the position measurements for the two beacons, with the left plot corresponding to $B_{1}$ and the right plot to $B_{2}$. It can be seen that the dispersion of the position is bigger for beacon $B_{2}$, with $\sigma_{x}=0.48 m$ and $\sigma_{y}=0.34 m$

the beacons are moored, they can nevertheless be affected by water current present. At the same time, it is known that positions obtained by GNSS have some intrinsic error that should be considered. Figure 5 plots the dispersion of the position measurements for the two beacons used, during the entire duration of the trial. While the dispersion of the measurements is different for the two beacons, this is probably caused by the position of each of the beacons. Nevertheless, the obtained standard deviation always remains below $0.5 \mathrm{~m}$, as indicated in the plots.

As previously mentioned, clock synchronization is essential for systems employing OWTT techniques. In the field trials, clock synchronization was achieved by using the PPS signals available from the GNSS receivers used. It was experimentally verified that by using this strategy synchronism between all the receivers can be achieved up to $25 \mathrm{~ns}$. Considering sound speeds of around $1500 \mathrm{~m} / \mathrm{s}$, this small difference in the PPS signals induces positions variations below the millimetre scale, which are considered to be negligible for the present application. Additionally, an implementation of NTP was set up in order to ensure clock synchrony and stability throughout the trials in the event of temporary failure of the GNSS receivers.

\subsection{Range measurements}

Both the ASVs used in this field trials, but also the beacons, are equipped with acoustic transducers that remain underwater, and can emit and detect acoustic signals in a predefined frequency range. The transducers are controlled by a proprietary electronic acoustic boards, in Fig. 6, and are linked to the PPS signal of the GNSS receptors, which allows them to be synchronized between each other.

The acoustic boards, mentioned above, are responsible to emit the acoustic signals and precisely time the detection of them. Then, the OWTT of the acoustic signals has to be con- 


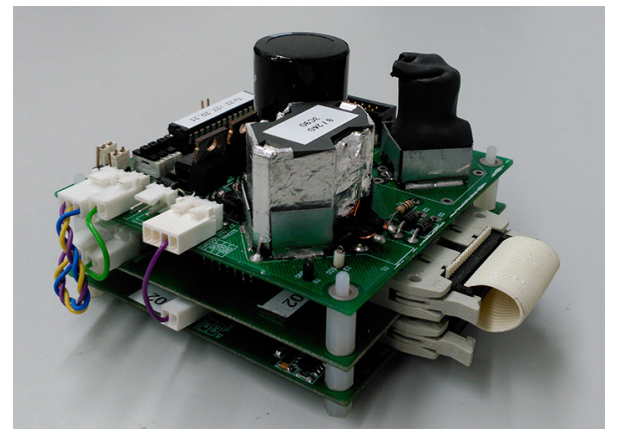

Fig. 6 Acoustic boards responsible for controlling the emission and detection of the acoustic signals

verted to ranges, provided that the speed of sound in the area of operations is known. Prior to the experimental validation here detailed, the necessary procedures to estimate an accurate speed of sound in the vicinity of the area of operations was performed. Because such procedure is not within the scope of this article, it won't be presented here for the sake of brevity, and the interested reader should refer to (Almeida et al. 2016). However, it should be noted that the speed of sound was assumed to be locally homogeneous. This means that the speed of sound was considered to be constant in the whole area of operations. Furthermore, it was also assumed than the slant ranges obtained by this method correspond directly to a distance on the horizontal plane. This is, in fact, approximately true, as the transducers of all the devices were mounted to be approximately all at the same depth.

In the field trials described in this section, two buoys with acoustic transceivers were used to detect the acoustic signals emitted by the vehicles. A set of slant range measurements collected by these buoys, from this point onwards referred to $B_{1}$ and $B_{2}$, are depicted in Fig. 7. The ranges correspond to the acoustic signals emitted by two distinct vehicles, corresponding to the blue and red colours in the figure. This clear distinction between the signals was achieved by having the two vehicles emitting signals in different frequencies, and was used only for a better data analysis and processing. Despite that, it should be noted that for the remaining of the analysis, the data used in the filter was stripped from any identifier that could potentially identify the origin of any of the signals.

In Fig. 7 the red and blue points correspond, respectively, to the ranges obtained by $B_{1}$ and $B_{2}$ originating from each of the vehicles. These ranges are the actual data used in the tracker for estimating the position of the two vehicles. It is clear from the figure that there is a continuous trend line for each of the vehicles, corresponding to their actual trajectories. However, it can also be observed that a high number of clutter measurements have been observer, particularly by $B_{2}$. Such outlier points are expected and common when dealing with underwater acoustic signals, and they arise from mul-
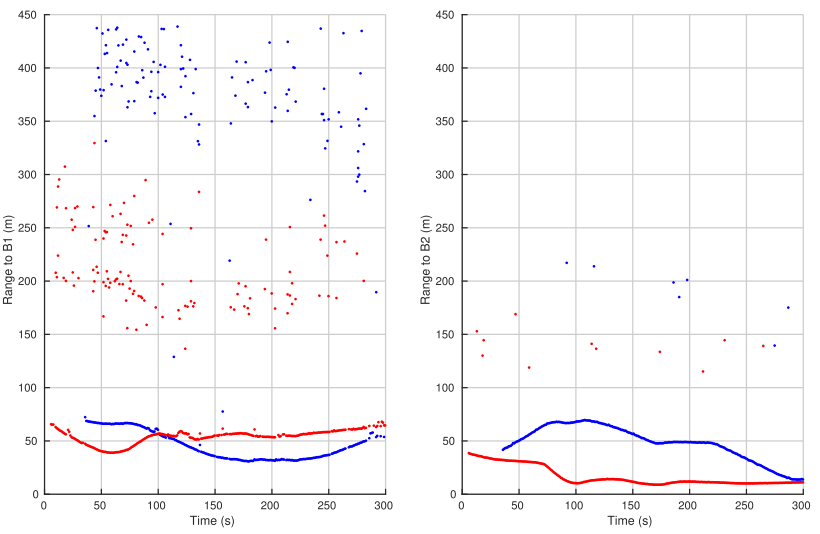

Fig. 7 Range measurements between two moving acoustic sources and two stationary beacons, $B_{1}$ and $B_{2}$, respectively on the left and right plots. The different colours correspond to acoustic ranges originating from different targets. It is clear that $B_{1}$ is detecting a lot more acoustic signals reflections

Table 1 Parameters of the multiple AUV tracker

\begin{tabular}{ll}
\hline Parameter & Value \\
\hline Filter settings & \\
Particles per target (N) & 1000 \\
Particles per birth $(\mathrm{M})$ & 1000 \\
Predictor settings & \\
Prob. of survival $\left(p_{s}\right)$ & 0.99 \\
Prob. of birth $\left(p_{b}\right)$ & $10^{-3}$ \\
Process noise variance $\left(\sigma_{x}^{2}, \sigma_{y}^{2}\right)$ & $0.5 \mathrm{~m}$ \\
Corrector settings & \\
Prob. of detection $\left(p_{d}\right)$ & 0.6 \\
Measurement noise variance $\left(\sigma_{r, B}^{2}\right)$ & $0.85 \mathrm{~m}$ \\
Clutter intensity $\left(\lambda_{k}\right)$ & $10^{-4} \sqrt{\left.\left|\mathbf{Z}_{k}\right|\right)} \mathrm{m}^{-2}$ \\
\hline
\end{tabular}

tipath phenomenons that affect acoustic signals, and their multiple reflections on either the bottom and the surface, or the margins. The fact that $B_{1}$ detects a lot more reflections than $B_{2}$, can probably find an explanation on the geometrical configuration of the setup.

\section{Results}

The main goal of the proposed tracker is to be able to estimate, in real-time, the position of multiple vehicles based on the acoustic ranges between each of the vehicles and a set of buoys, or acoustic beacons. The tracking results were obtained by using the collected set of range measurements, shown above, to the multiple AUV tracker derived in the previous sections. The different parameters of the tracker used to 
Fig. 8 Overview of the mission: trajectories of the vehicles and position of the beacons. It is also shown the area where new targets are expected to appear
Fig. 9 Time evolution of the position of the targets, in blue and red, respectively. Dashed lines correspond the ground truth
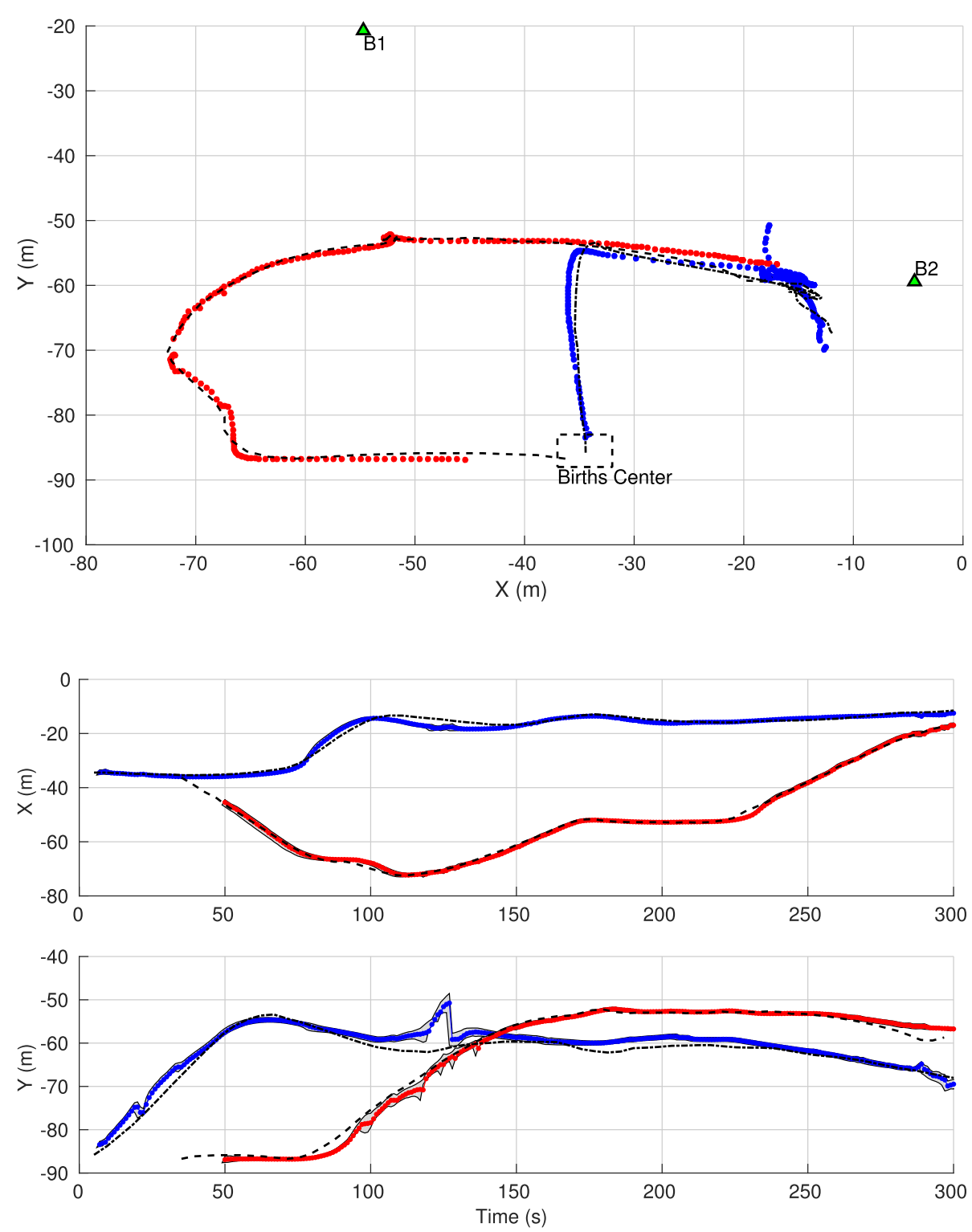

obtain the results that are going to be presented are specified in Table 1.

One of the missions performed for the experimental evaluation of the tracker, and the one that will be here presented, consisted on having two vehicles navigating simultaneously in a predefined area, under surveillance of two moored acoustic beacons. We had the vehicles starting to emit acoustic signals at different times, in order to illustrate the ability of the filter to detect new vehicles entering the surveillance area. At the same time, the vehicles were navigating with arbitrary varying velocities and in different directions, but also being in stationary. An overview of one of the trajectories performed by each of the vehicles can be seen in Fig. 9 .

There we can see the position of each of the buoys, marked with $B_{1}$ and $B_{2}$, together with the trajectories performed by the two vehicles, the real ones, provided by GNSS, in dashed black line, and the estimated ones in blue and red, respectively. The area where new targets are expected to be birthed is also marked.

For a better understanding of the accuracy of the position estimations we present, in Fig. 9 the time evolution of the estimated positions of the two targets, and compare it with the ground truth, given by the GNSS position of the targets. Besides the estimated position, the plot also includes the standard deviation of the the estimation. It can be seen that the estimated trajectories of the vehicle closely resemble the trajectories given by the ground truth data.

A closer look into Fig. 9 reveals that between seconds $t=120 \mathrm{~s}$ and $t=130 \mathrm{~s}$, approximately, the position of the target in blue colour diverges from the ground truth for some time, but then quickly recovers. This behaviours is caused by a situation on which the ranges received by one of the 
Fig. 10 Illustration of a situation where the deghosting algorithm successfully eliminates ghost targets. Red and blue indicate the two different targets being tracked, with the black dots corresponding to particles assigned to new-born targets. a t $=124 \mathrm{~s}$, (b) $\mathrm{t}=125 \mathrm{~s}, \mathbf{c t}=126 \mathrm{~s}$, $\mathbf{d} \mathrm{t}=127 \mathrm{~s}, \mathbf{e} \mathrm{t}=128 \mathrm{~s}, \mathbf{f}$ $\mathrm{t}=129 \mathrm{~s}$

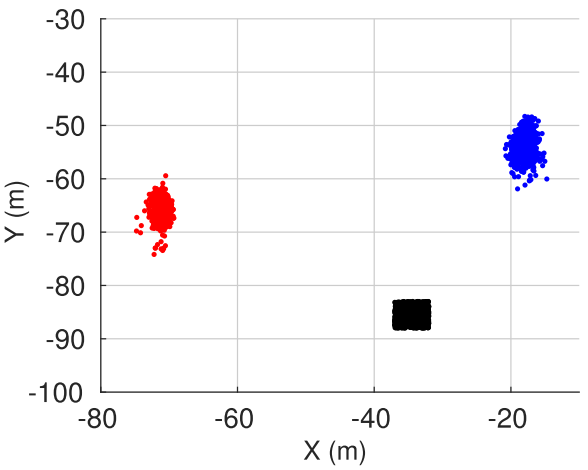

(a)

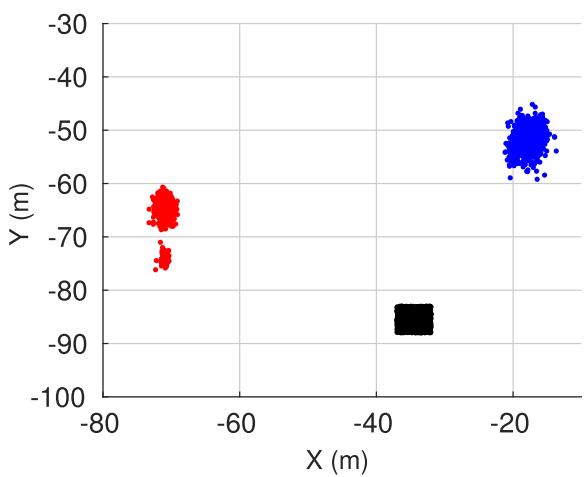

(c)

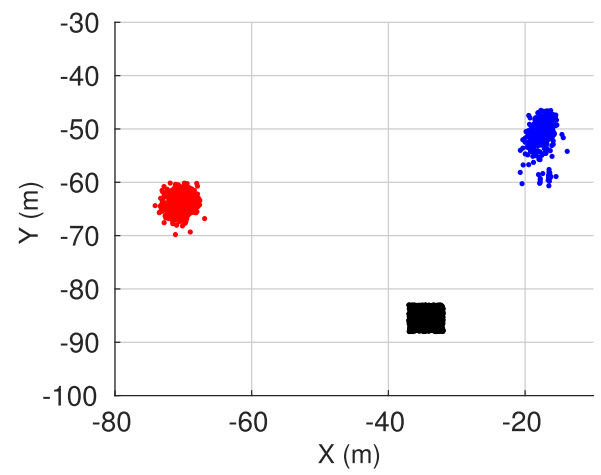

(e)

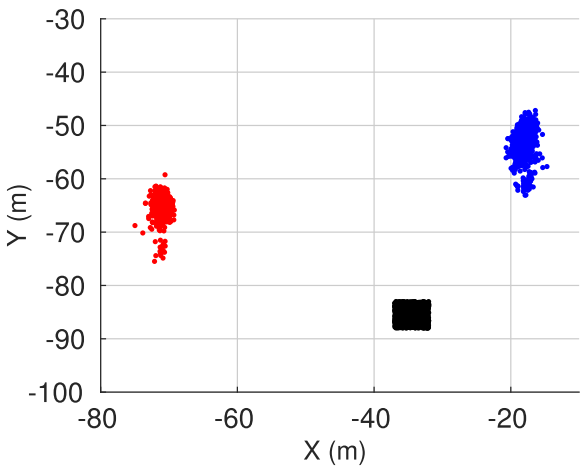

(b)

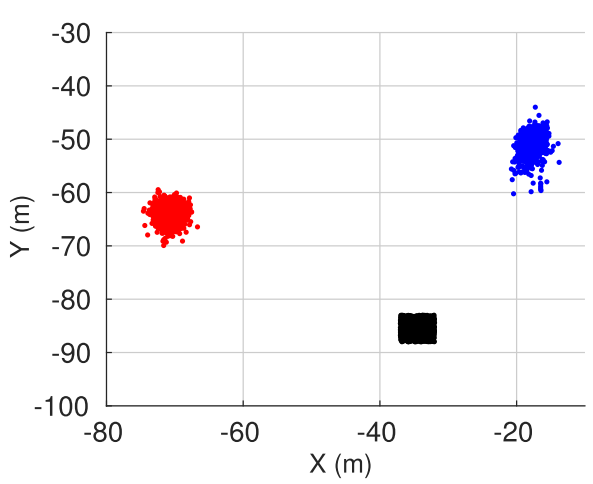

(d)

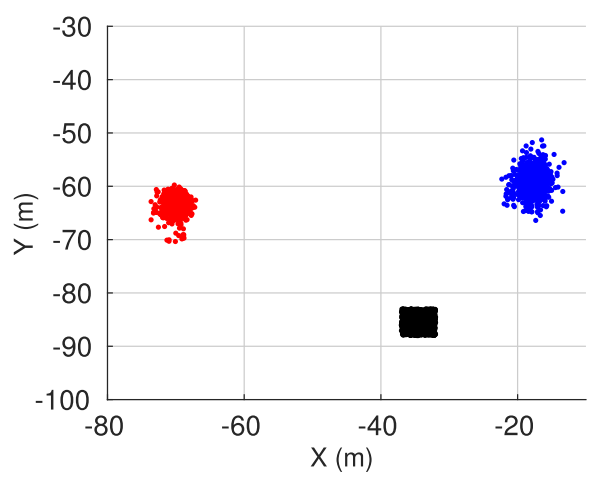

(f) beacons, in this case $B_{1}$, from both the vehicles have similar magnitude. This corresponds to a situation where both vehicles are at equal distances to one of the buoys, and configures a case on which ghost targets are likely to arise, as described in the previous section. Figure 10 details the behaviour of the filter in that situation, by showing the distribution of the particles tracking each of the vehicles.

The sequence in Fig. 10 illustrates the behaviour of the developed deghosting heuristics, preventing the creation of a new ghost target. At $t=124 \mathrm{~s}$, in Fig. 10a, the particles that track each of the targets are mildly concentrated around the actual position of the targets. In Fig. 10b, c) it can be seen that the particles corresponding to each of the targets start to be less concentrated and more spread in space. It can even be perceived two different clusters of particles being formed in the vicinity of each of the targets. The deghosting strategy implemented prevents the creation of ghost targets, and in Fig. 10d-f the success of such strategy can be observed, with the two apparent clusters becoming only one.

Figures 8, 9 and 10 demonstrate the good tracking performance of our Multiple AUV Tracker, as it is easy to see that the estimated positions of both the targets closely resemble the ground-truth GPS data, with their differences being well within the expected. The obtained root mean square (RMS) errors for the entire duration of this experiment were, respectively of $1.98 \mathrm{~m}$ for the target in blue, and $1.18 \mathrm{~m}$ for the target in red. Figure 11 shows the absolute error in position between estimated and ground truth positions. 
Fig. 11 Absolute errors between ground truth and estimated positions
Fig. 12 Velocities of the two targets, in blue and red, respectively. Dashed lines correspond to velocities provided by the onboard navigation systems of the vehicles (Color figure online)
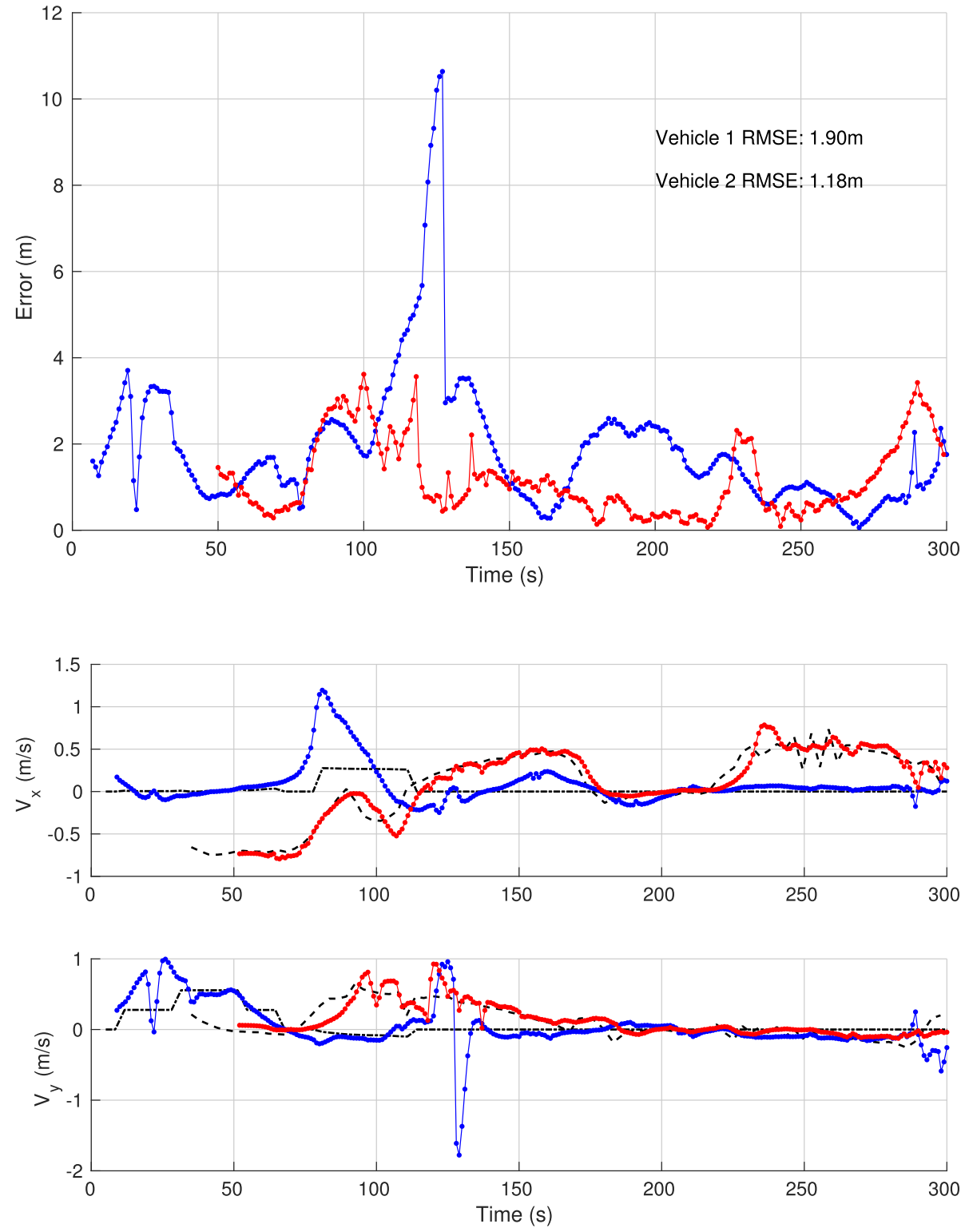

By analysing Fig. 11, it can be concluded that the absolute error in position stays well below the $2 \mathrm{~m}$ for several occasions, which corresponds to situations where the targets move in straight lines. Conversely, when the vehicles change direction, this error increases. The situation depicted in Fig. 10, when the occurrence of a ghost target is prevented, has obviously repercussions on the error in tracking the vehicle, which corresponds to the peak present in Fig. 11, with the error being of around $10 \mathrm{~m}$ for one of the targets. Nevertheless, it can also be observed that as the tracker recovers from this situation, also the tracking error decreases. It should be noted that RMS errors for both the vehicles is comparable to the precision obtained by common GPS receivers while operation in single mode precision.
Finally, we present a comparison between the estimated velocities of each of the targets, and the velocities provided by the navigation layer of each of the vehicles. While this is an interesting comparison for the sake of the analysis of the achievable performance of the tracker, it should be noted that the velocities available from the on-board software of the vehicles can't be understood as ground truth. In fact, such values result from fusing together single mode GNSS position and IMU data and might not reflect the exact speed of the vehicles. Nevertheless, as it can be seen in Fig. 12, the speed profiles are moderately similar, specially for the red target. However, in some situations it seems that the estimated velocity lags in time. which is probably caused by the RLSE, and dependent on its window size. As before, the velocities estimated by the tracker are more close to the ones provided 
by the navigation layer when the vehicles are moving in for straight-line.

\section{Conclusion}

Operations with multiple vehicles are likely to become very appealing in a near future, not only in terms of flexibility and efficiency, but also in terms of performing a set task that otherwise would not be possible. While the problem of providing navigational aids to multiple vehicles has been addressed in the past, tracking multiple AUVs is a problem that has been overlooked. In this article we presented an effective AUV Tracker for simultaneously tracking multiple vehicles, and successfully demonstrated its performance for real world scenarios. Up to the authors knowledge, this is the first time that a similar approach has been proposed and experimentally validated. Moreover, a similar strategy can be applied to related applications, such as the localization and tracking of multiple underwater acoustic sources.

It was demonstrated that the problem of tracking of multiple AUVs can be successfully tackled using PHD filters. The RFS nature of the PHD filter doesn't require a specific association between measurements and the targets that produced them, thus making it appropriate for this problem. Also, with this strategy there is no need to develop intricate solutions to distinguish vehicles, such as complex hardware for modulating the acoustic signals. The tracking results achieved were very positive, with RMS errors under 2 meters, which is of similar precision to what can be obtained with a GPS receiver. Moreover, the proposed filter is also able to keep information of the track continuity along the time. Such results were obtained despite the quite adverse trajectories performed by the vehicles, very close to each other, and with the vehicles moving with arbitrary low speeds. It should also be underlined that such results have been achieved despite the unfavourable geometry of the acoustic network, with only two beacons and with a baseline not very long.

While the results achieved are very encouraging, it can be argued that we addressed a situation with only two targets. While this is true, there is no indication that the developed tracker would have behaved differently with more targets appearing and disappearing in the area under surveillance. While the computational complexity of the filter depends exponentially on the number of clutter measurements, and this number would obviously increase with the number of targets being tracked, the implementation of an adequate gating strategy, as it has been proposed elsewhere, is able to properly deal with this issue. On the other hand, the processing time of the algorithm, for the full duration of the scenario in analysis, is well below the actual time, thus no real-time performance issues are likely to arise.
As a final remark, it should be mentioned that even though the proposed approached was focused on tracking AUVs, it's applicability can be broader. As it was made clear in Sect. 2, the topic of underwater target tracking is broader and encompasses more areas than just the underwater robotics community. Therefore, it would be interesting to study the applicability of the proposed method to other areas, for example related to underwater wireless sensor networks, or even source localization problems.

Acknowledgements This work is financed by the ERDF-European Regional Development Fund through the Operational Programme for Competitiveness and Internationalisation - COMPETE 2020 Programme, and by National Funds through the FCT-Fundação para a Ciência e a Tecnologia (Portuguese Foundation for Science and Technology) within project «POCI-01-0145-FEDER-006961 ». The first author was supported by the Portuguese Foundation for Science and Technology through the Ph.D. grant SFRH/BD/70727/2010.

\section{Compliance with ethical standards}

Conflicts of interest The authors declare that they have no conflict of interest.

\section{References}

Almeida, R., Melo, J., \& Cruz, N. (2016). Characterization of measurement errors in a LBL positioning system. In Proceedings of the MTS/IEEE Oceans'16 Conference, Shanghai, China.

Bar-Shalom, Y., \& Li, X. (1995). Multitarget-multisensor tracking: Principles and techniques. London: Yaakov Bar-Shalom.

Braca, P., Goldhahn, R., LePage, K., Marano, S., Matta, V., \& Willett, P. (2014). Cognitive multistatic AUV networks. In 2014 17th international conference on information fusion (FUSION) (pp. 1-7).

Choi, J. \& Choi, H. -T. (2015). Multi-target localization of underwater acoustic sources based on probabilistic estimation of direction angle. In Proceedings of the MTS/IEEE Oceans'15 conference, Genova, Italy (pp. 1-6).

Clark, D., \& Bell, J. (2007). Multi-target state estimation and track continuity for the particle phd filter. IEEE Transactions on Aerospace and Electronic Systems, 43(4), 1441-1453.

Clark, D. E. (2006). Multiple target tracking with the probability hypothesis density filter. Ph.D. thesis, Heriot-Watt University.

Cruz, N., Matos, A., Cunha, S., \& Silva, S. (2007). Zarco-An autonomous craft for underwater surveys. In Proceedings of the 7th geomatic week, Barcelona, Spain.

Erdinc, O., Willett, P., \& Coraluppi, S. (2008). The gaussian mixture cardinalized phd tracker on mstwg and seabar datasets. In Information Fusion, 2008 11th International Conference on, pages 1-8.

Fiorelli, E., Leonard, N., Bhatta, P., Paley, D., Bachmayer, R., \& Fratantoni, D. (2006). Multi-auv control and adaptive sampling in monterey bay. IEEE Journal of Oceanic Engineering, 31(4), 935-948.

Fulton, T. F., Cassidy, C. J., Stokey, R. G., \& Leonard, J. J. (2000). Navigation sensor data fusion for the AUV REMUS. In Proceedings of the symposium on underwater robotic technology, World Automation Congress, Hawaii.

Georgescu, R., Schoenecker, S., \& Willett, P. (2009). Gm-cphd and mlpda applied to the seabar07 and tno-blind multi-static sonar data. 
In 2009. FUSION '09. 12th international conference on information fusion (pp. 1851-1858).

Georgy, J., \& Noureldin, A. (2014). Unconstrained underwater multitarget tracking in passive sonar systems using two-stage pf-based technique. International Journal of Systems Science, 45(3), 439455.

Georgy, J., Noureldin, A., \& Mellema, G. (2012). Clustered mixture particle filter for underwater multitarget tracking in multistatic active sonobuoy systems. IEEE Transactions on Systems, Man, and Cybernetics, Part C: Applications and Reviews, 42(4), 547560 .

Granstrom, K., Lundquist, C., Gustafsson, F., \& Orguner, U. (2014). Random set methods: Estimation of multiple extended objects. IEEE Robotics and Automation Magazine, 21(2), 73-82.

Hol, J., Schon, T., \& Gustafsson, F. (2006). On resampling algorithms for particle filters. In 2006 IEEE nonlinear statistical signal processing workshop (pp. 79-82).

Kreucher, C., \& Shapo, B. (2011). Multitarget detection and tracking using multisensor passive acoustic data. IEEE Journal of Oceanic Engineering, 36(2), 205-218.

Lang, T., Dunne, D., \& Mellema, G. (2009). An assessment of hierarchical data fusion using seabar'07 data. In 2009. FUSION '09. 12th international conference on information fusion (pp. 1560-1567).

Leonard, N. E., Paley, D. A., Davis, R. E., Fratantoni, D. M., Lekien, F., \& Zhang, F. (2010). Coordinated control of an underwater glider fleet in an adaptive ocean sampling field experiment in monterey bay. Journal of Field Robotics, 27(6), 718-740.

Lin, L., Bar-Shalom, Y., \& Kirubarajan, T. (2006). Track labeling and phd filter for multitarget tracking. IEEE Transactions on Aerospace and Electronic Systems, 42(3), 778-795.

Ma, W.-K., Vo, B.-N., Singh, S., \& Baddeley, A. (2006). Tracking an unknown time-varying number of speakers using tdoa measurements: A random finite set approach. IEEE Transactions on Signal Processing, 54(9), 3291-3304.

Mahler, R. (2003). Multitarget bayes filtering via first-order multitarget moments. IEEE Transactions on Aerospace and Electronic Systems, 39(4), 1152-1178.

Mahler, R. (2008). Random set theory for multisource-multitarget information fusion. In M. E. Liggins, D. L. Hall, \& J. Llinas (Eds.), Handbook of multisensor data fusion: Theory and practice (second ed., pp. 369-410). Boca Raton: CRC Press.

Mahler, R. (2013). "statistics 102" for multisource-multitarget detection and tracking. IEEE Journal of Selected Topics in Signal Processing, 7(3), 376-389.

Marino, A., Antonelli, G., Aguiar, A., Pascoal, A., \& Chiaverini, S. (2015). A decentralized strategy for multirobot sampling/patrolling: Theory and experiments. IEEE Transactions on Control Systems Technology, 23(1), 313-322.

Matos, A., Cruz, N., Martins, A., \& Pereira, F. (1999). Development and implementation of a low-cost lbl navigation system for an auv. In Proceedings of the MTS/IEEE Oceans'99 conference (Vol. 2, pp. 774-779). Seattle, USA.

Mazurek, P. (2008). Deghosting methods for track-before-detect multitarget multisensor algorithms. In J. M. R. Arreguin (Ed.), Automation and Robotics. I-Tech Education and Publishing.

Melo, J., \& Matos, A. (2008). Guidance and control of an asv in auv tracking operations. In Proceedings of the MTS/IEEE Oceans'08 conference (pp. 1-7), Quebec City, Canada.

Melo, J., \& Matos, A. (2014). A phd filter for tracking multiple auvs. In Proceedings of the MTS/IEEE Oceans'14 conference (pp. 1-8), St. John's, Canada.
Morelande, M., Suvorova, S., Fletcher, F., Simakov, S., \& Moran, B. (2015). Multi-target tracking for multistatic sonobuoy systems. In 2015 18th International Conference on Information Fusion (Fusion) (pp. 327-332).

Napolitano, F., Cretollier, F., \& Pelletier, H. (2005). Gaps, combined usbl + ins + gps tracking system for fast deployable amp; high accuracy multiple target positioning. In Proceedings of the MTS/IEEE Oceans'08 conference (Vol. 2, pp. 1415-1420), Brest, France.

Odell, D., Hertel, K., \& Nielsen, C. (2002). New acoustic systems for auv tracking, communications, and noise measurement at nswccdard, lake pend oreille, idaho. In Proceedings of the MTS/IEEE Oceans'02 conference (Vol. 1, pp. 266-271), Biloxi, USA.

Paull, L., Huang, G., Seto, M., \& Leonard, J. (2015). Communicationconstrained multi-auv cooperative slam. In 2015 IEEE international conference on robotics and automation (ICRA) (pp. 509-516).

Peñas, A. A. (2009). Positioning and navigation systems for robotic underwater vehicles. Ph.D. thesis, Instituto Superior Técnico, Universidade Técnica de Lisboa.

Prins, R., \& Kandemir, M. (2008). Time-constrained optimization of multi-auv cooperative mine detection. In Proceedings of the MTS/IEEE Oceans'08 conference (pp. 1-13), Quebec City, Canada.

Schulz, B., Hobson, B., Kemp, M., Meyer, J., Moody, R., Pinnix, H., $\&$ St Clair, M. (2003). Field results of multi-uuv missions using ranger micro-uuvs. In Proceedings of the MTS/IEEE Oceans'03 conference (Vol. 2, pp. 956-961), San Diego, USA.

Stone, L., Streit, R., Corwin, T., \& Bell, K. (2013). Bayesian multiple target tracking, Second Edition: Radar/Remote Sensing. Artech House.

Vahidi, A., Stefanopoulou, A., \& Peng, H. (2005). Recursive least squares with forgetting for online estimation of vehicle mass and road grade: Theory and experiments. Vehicle System Dynamics, 43(1), 31-55.

Vo, B. N., \& Ma, W. K. (2005). A closed-form solution for the probability hypothesis density filter. In 7th international conference on information fusion (Vol. 2, p. 8).

Vo, B.-N., Singh, S., \& Doucet, A. (2005). Sequential monte carlo methods for multitarget filtering with random finite sets. IEEE Transactions on Aerospace and Electronic Systems, 41(4), 12241245.

Vo, B.-T., \& Vo, B.-N. (2013). Labeled random finite sets and multiobject conjugate priors. IEEE Transactions on Signal Processing, 61(13), 3460-3475.

Wang, X., Xu, M., Wang, H., Wu, Y., \& Shi, H. (2012). Combination of interacting multiple models with the particle filter for three-dimensional target tracking in underwater wireless sensor networks. Mathematical Problems in Engineering, 2012(829451), $1-16$.

Watanabe, Y., Ochi, H., Shimura, T., \& Hattori, T. (2009). A tracking of auv with integration of ssbl acoustic positioning and transmitted ins data. In Proceedings of the MTS/IEEE Oceans'09 conference (pp. 1-6), Bremen, Germany.

Yang, R., Ng, G. W., \& Bar-Shalom, Y. (2013). Tracking/fusion and deghosting with doppler frequency from two passive acoustic sensors. In 2013 16th international conference on information fusion (FUSION) (pp. 1784-1790).

Zhang, S., Chen, H., \& Liu, M. (2014). Adaptive sensor scheduling for target tracking in underwater wireless sensor networks. In 2014 international conference on mechatronics and control (ICMC) (pp. $55-60)$. 


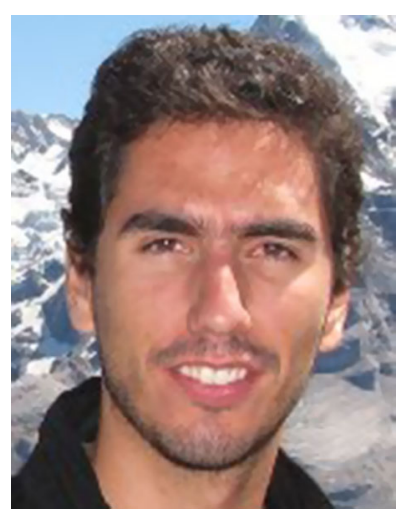

José Melo is a postdoc researcher at the Center for Robotics and Autonomous Systems of INESCTEC, in Porto. In 2016 he received his Ph.D. in Electrical and Computer Engineering from the Faculty of Engineering, University of Porto. In 2008 he received his M.Sc. degree in Electrical and Computer Engineering, with a major in Automation, from the same University. In between degrees, and until 2010, José has worked as a Software Engineer, at CERN. José is with the Ocean Systems Group since 2010, when he started his Ph.D., and with INESCTEC since 2011, when he joined the Robotics and Intelligent Systems unit as a researcher. In 2015 he was an invited researcher at the National Insitute of Informatics, Tokyo, Japan under the supervision of Prof. Kenki Tei, in Honiden Lab. His current research interests are in the area of Navigation and Sensor Fusion for Autonomous Vehicles.

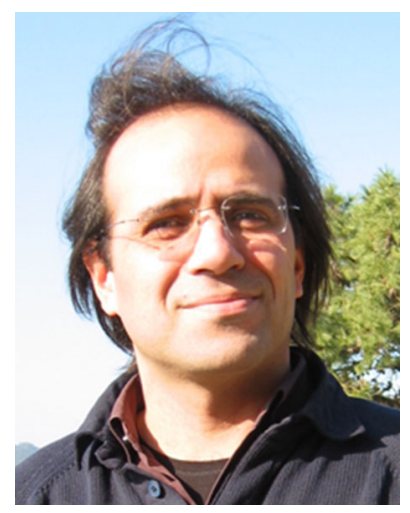

Aníbal C. Matos received the $\mathrm{Ph} . \mathrm{D}$. degree in Electrical and Computer Engineering from the University of Porto, Porto, Portugal, in 2001. Currently, he coordinates de Centre for Robotics and Autonomous Systems at INESC TEC and holds an assistant professor position at the Faculty of Engineering of the University of Porto. His main research interests are related to navigation and control of autonomous underwater and surface vehicles. He has been and is currently involved in several $R \& D$ projects related to the development and applications of marine vehicles, funded by several national and international agencies. 